\title{
Cellular birthdate predicts laminar and regional cholinergic projection topography in the forebrain.
}

Kathryn C. Allaway ${ }^{1,2,3}$, William Muñoz ${ }^{1}$, Robin Tremblay ${ }^{1}$, Mia Sherer ${ }^{2,3,4}$, Jacob Herron $^{2,3,4}$, Bernardo Rudy ${ }^{1}$, Robert Machold ${ }^{1 *}$, Gord Fishell ${ }^{2,3 *}$

${ }^{1}$ Neuroscience Institute, New York University, New York, New York 10016, USA

${ }^{2}$ Department of Neurobiology, Harvard Medical School, Boston, MA 02115, USA.

${ }^{3}$ Stanley Center for Psychiatric Research, Broad Institute, Cambridge, MA 02142, USA.

${ }^{4}$ Northeastern University, Boston, MA, 02115, USA

${ }^{*}$ Co-corresponding authors 


\section{Abstract}

2 The basal forebrain cholinergic system projects broadly throughout the cortex and

3 constitutes a critical source of neuromodulation for arousal and attention. Traditionally,

4 this system was thought to function diffusely. However, recent studies have revealed a

5 high degree of spatiotemporal specificity in cholinergic signaling. How the organization

6 of cholinergic afferents confers this level of precision remains unknown. Here, using

7 intersectional genetic fate mapping, we demonstrate that cholinergic fibers within the

8 cortex exhibit remarkable laminar and regional specificity and that this is organized in

9 accordance with cellular birthdate. Strikingly, birthdated cholinergic projections within

10 the cortex follow an inside-out pattern of innervation. While early born cholinergic

11 populations target deep layers, late born ones innervate superficial laminae. We also

12 find that birthdate predicts cholinergic innervation patterns within the amygdala,

13 hippocampus, and prefrontal cortex. Our work reveals previously unappreciated

14 specificity within the cholinergic system and the developmental logic by which these

15 circuits are assembled. 
Introduction

18 Acetylcholine (ACh) plays an essential role in modulating attention, motivation, and

19 learning within neocortical, hippocampal, and subcortical circuits (Ballinger et al., 2016;

20 Luchicchi et al., 2014; Picciotto et al., 2012). The primary source of ACh within these

21 structures are the projection neurons located in several nuclei throughout the basal

22 forebrain. Historically, these neurons were thought to be relatively indiscriminate sources

23 of ACh, releasing it slowly and diffusely throughout the cortex to mediate widespread

24 circuit activation (Sarter et al., 2009). Nonetheless, considerable in vitro work has implied

25 a multitude of layer-, cell type-, and synapse-specific cholinergic effects (reviewed in

26 Muñoz and Rudy, 2014). Recent in vivo findings have revealed an even higher level of

27 spatiotemporal coordination (Froemke et al., 2007; Muñoz et al., 2017). These

28 observations hint that the ACh system is composed of distinct cell types that target

29 specific layers and circuit elements of the cortex in a temporally precise manner.

31 Classically, the basal forebrain cholinergic neurons (BFCNs) have been divided into four

32 groups based upon cell body location - Ch1 (medial septum), Ch2 (vertical diagonal

33 band), Ch3 (horizontal diagonal band), and Ch4 (substantia innominata and nucleus

34 basalis) (Mesulam et al., 1983). These broad anatomical divisions roughly reflect their

35 innervation of different brain structures (i.e., neocortex, hippocampus, and amygdala).

36 Further efforts delineated a more refined topography. Early work suggested that rostrally

37 located cells project to medial cortical areas and that caudal cells project laterally

38 (Baskerville et al., 1993; Saper, 1984). This work was primarily carried out using 
39 anterograde and retrograde tracers which allowed the broad spatial topography to be

40 determined, but could not distinguish between cholinergic and noncholinergic cells.

41 More recently, genetic tools have allowed for the sparse, selective targeting of

42 cholinergic cell types but failed to reveal their overall topographic and anatomical

43 organization (Li et al., 2018; Wu et al., 2014; Zaborszky et al., 2015). Here, by using a

44 hybrid genetic/retrograde labeling strategy, we demonstrate the existence of cholinergic

45 neurons with specific projections to deep, middle, and superficial layers of the mouse

46 somatosensory cortex.

48 We further sought to understand how this organization in the cholinergic system arises

49 during development. It is known that all cholinergic neurons in the forebrain originate 50 within the Nkx2.1+ proliferative region of the ventral embryonic telencephalon (Marin et

51 al., 2000; Patel et al., 2012; Xu et al., 2008). The diversity of other cell types arising from

52 this region is generated by both spatially defined progenitor pools (Gelman et al., 2009;

53 Nóbrega-Pereira et al., 2010; Wonders et al., 2008), as well as temporal shifts in the

54 neuronal subtype produced (Inan et al., 2012; Miyoshi et al., 2007). Here, we investigated

55 the developmental origins of cholinergic projection neuron topography. We were

56 surprised to find that the temporal, but not the spatial, organization of the progenitors

57 predicted the organization of the mature cholinergic projections. Furthermore, we find

58 that the axons of cholinergic neurons born at different embryonic timepoints take distinct

59 pathways to reach their projection targets. Together, these results illustrate that the

60 temporal origins of the BFCNs predict the precise organization of their cortical and

61 subcortical axonal topographies. 


\section{Results}

Layer-specific cholinergic projections in the mature somatosensory cortex

67 To explore specificity of cholinergic axonal arborizations, we used a hybrid genetic/viral

68 strategy to label small subpopulations of cholinergic neurons innervating the mature

69 cortex. To that end, we generated a mouse line in which the FlpO recombinase was

70 targeted to the choline acetyltransferase locus $\left(C h A T^{F / p O}\right)$. When used in the context of

71 an Flp-dependent reporter, this enables the specific labelling of cholinergic neurons.

72 This mouse line exhibits the expected selectivity of FlpO expression within cholinergic

73 neurons when crossed to a pan-ventral $\left(D / \times 6 a^{C r e}\right)$ line and visualized using an

74 intersectional reporter (Ai65) (Figure 1 - figure supplement 1).

75

76 To determine whether cholinergic neurons have restricted arborizations within specific

77 layers of the somatosensory cortex, we crossed the ChAT Fipo line to the Ai65

78 intersectional (i.e., Cre and Flp dependent) reporter allele and injected these animals with

79 a Cre-expressing type 2 canine adenovirus (CAV-2::Cre). This virus specifically infects

80 axon terminals (Ekstrand et al., 2014; Junyent and Kremer, 2015), resulting in Cre

81 expression only in cells that innervate the injected region. By restricting this virus to

82 either the superficial or deep layers of the primary somatosensory cortex of $C h A T^{F / p O} ; A i 65$

83 mice (Figure 1A), we were able to selectively label cholinergic neurons with axons

84 projecting to specific cortical layers. The fluorescent reporter fills the entire axonal 
85 arborization of labeled cholinergic neurons and thus allowed us to determine the extent

86 of arborization of individual cells across laminae. Using this method, we observed that

87 the majority of projections were restricted to the injected layers. This indicates that most

88 cholinergic neurons predominantly arborize within specific cortical laminae (Figure 1B,

89 C). In particular, deep layer injections largely showed projections restricted to layers 5

90 and 6 (L5-6) of the somatosensory cortex, whereas superficial injections primarily

91 showed projections within layers 1 through 4 (L1-4), with some showing remarkable

92 specificity for L1.

94 The layer-specificity of cholinergic projections was further confirmed by two high-

95 resolution, single-cell reconstructions of the axonal arbors of individually labeled

96 cholinergic neurons- one targeting L1 of the somatosensory cortex, and another

97 targeting L5-6 (Figure 1G, H; Figure 1- figure supplements 2 and 3). The soma of the L1-

98 targeting cell was located in the rostral substantia innominata, sending its axon rostrally

99 then dorsally through the medial septum, ultimately entering the cortex through L1.

100 Despite the fact that this cell had arborizations extending from the caudal motor cortex

101 to the rostral visual cortex, it was almost entirely restricted to layer 1. Conversely, the

102 soma of the L5/6-targeting cell was located in the nucleus basalis, near the globus

103 pallidus. Its axon traversed through the striatum to enter the external capsule before

104 entering the somatosensory cortex from L6. Unlike the L1-targeting cell, the

105 arborizations of the L5/6-targeting cell were restricted to the somatosensory cortex and 106 covered a much more limited rostral to caudal territory. 
108 Moreover, we mapped the soma locations of the labeled cholinergic neurons within the

109 basal forebrain in order to gain insight into whether cell body localization is related to the

110 layer topography of their axonal arborizations (Figure 1D, E, F). We found that both

111 deep- and superficial-targeting cholinergic neurons can reside within one of several

112 nuclei across the rostral-caudal axis of the basal forebrain, including the vertical diagonal

113 band (VDB), horizontal diagonal band (hDB), substantia innominata (SI), and nucleus

114 basalis (NB). We did note, however, that there is a bias for deep layer-projecting cells to

115 be predominantly located in more caudal structures (i.e. Bregma -2.0 to -0.5 ,

116 corresponding to the NB and caudal SI), while superficially-projecting cells are enriched

117 in more rostral BF structures (Bregma -0.5 to +0.5 , corresponding to the $\mathrm{hDB}$ and rostral

$118 \mathrm{SI}$ (ANOVA, $F(2,18)=13.63, p=0.0037)$. 

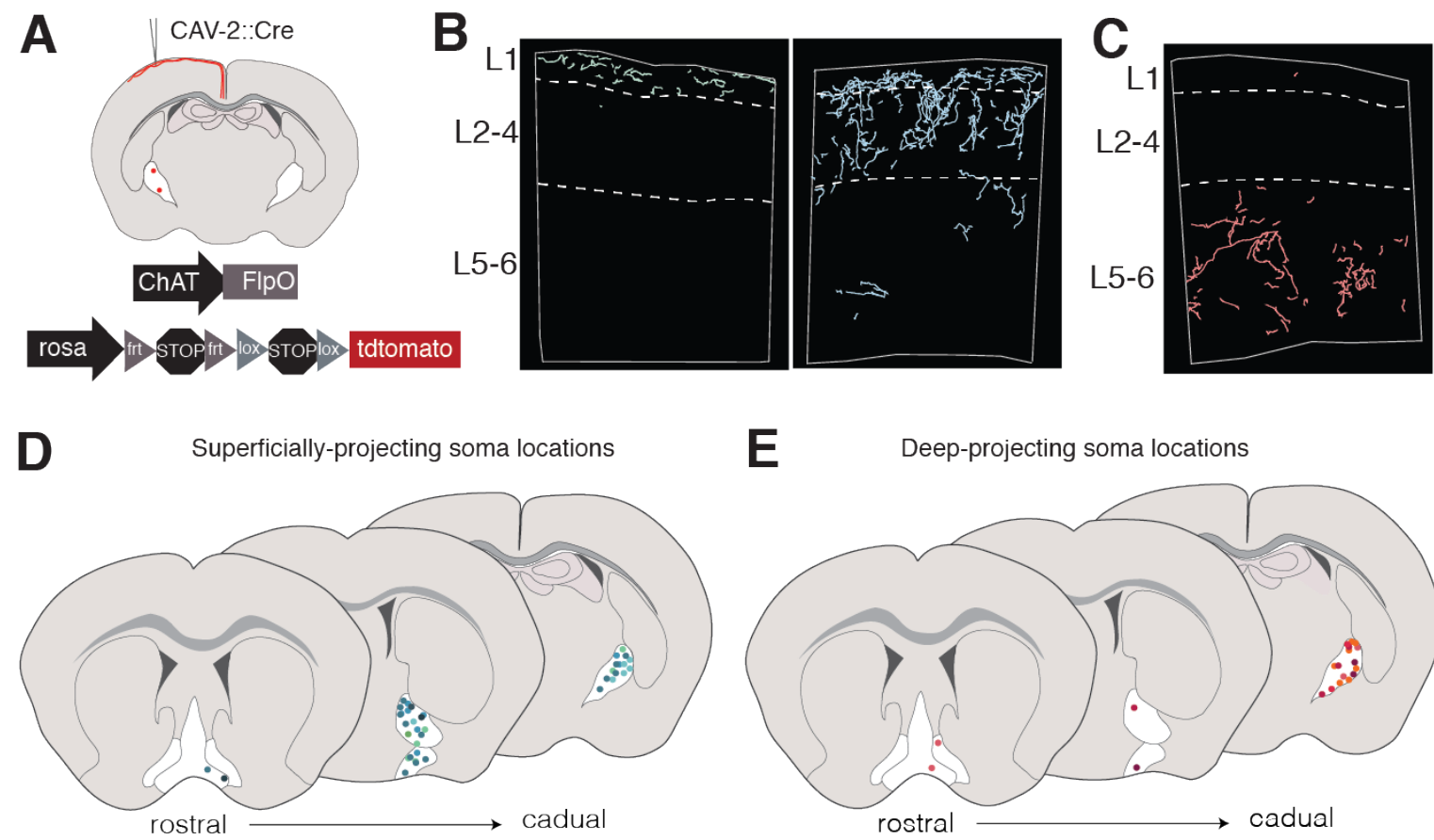

E Deep-projecting soma locations
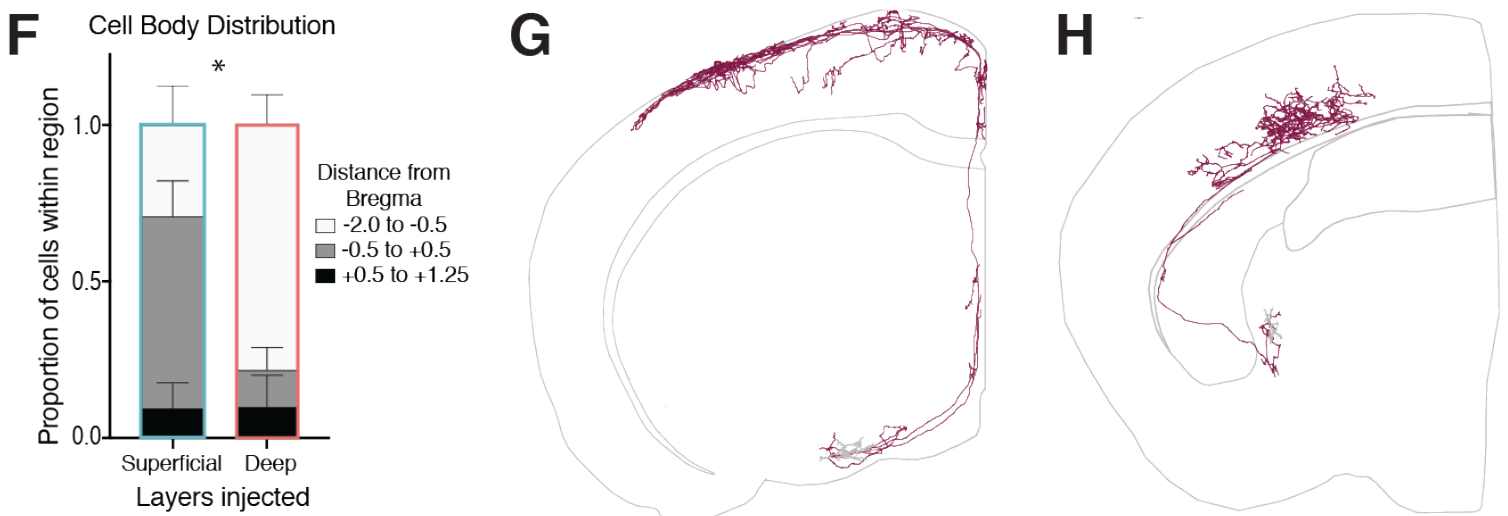

Figure 1. Retrograde labeling of layer-specific cholinergic projections in the somatosensory cortex. (A) Genetic and viral strategy for specific labeling of cholinergic efferents to superficial or deep layers of the somatosensory cortex. (B) Reconstructed fibers within representative sections of the somatosensory cortex from superficial layer injections, some of which were remarkably L1specific (left) and some which exhibited fibers in L1-4 (right). (C) Reconstructed fibers within representative sections from a deep layer injection. (D and E) Cell body locations within the basal forebrain of cholinergic neurons labeled in superficial- (D) or deep- (E) layer injections. Shades of colors within (D) and (E) represent individual animals (superficial injections $n=6$, deep injections $n=5$ ). (F) Proportions of labeled cell bodies located across the rostral to caudal axis in the basal forebrain. Proportions were calculated for each injection individually then averaged, error bars show SEM. Twoway ANOVA $F(2,18)=13.63, p=0.0037$; Sidak's multiple comparisons test shows the proportion of cells located between Bregma -0.5 and +0.5 is significantly different between groups $(p=0.0466)$. (G) Complete reconstruction of a cholinergic neuron with the majority of its axonal arborizations in L1. (H) Complete reconstruction of a cholinergic neuron with restricted projections in L5-6. Reconstructions are shown on a representative hemisphere from a coronal section. Red fibers indicate axonal projections, gray fibers indicate dendrites. 
Spatial embryonic origin does not predict projection patterns of cholinergic neurons

122 We then wanted to determine the developmental origins of these layer-specific

123 cholinergic neurons, both to understand how this layer specificity emerges and to 124 identify genetic tools that could be used to target these neurons for further investigation.

125 We first asked whether layer-specific cholinergic neurons arise from distinct spatial 126 locations within the ventral embryonic telencephalon. Embryonic progenitor zone origin

127 has been shown to predict subtype identity within other forebrain lineages (Bandler et 128 al., 2017; Lim et al., 2018), as well as projection neuron populations with distinct targets 129 in the hindbrain (Jensen et al., 2008; Robertson et al., 2013, 2016).

131 Previous work has shown that all BFCNs arise from the Nkx2.1+ domain within the

132 ventral telencephalon, which encompasses both the medial ganglionic eminence (MGE)

133 and preoptic area (POA). These two regions have distinct patterns of gene expression

134 (Flames et al., 2007; Hansen et al., 2013) and have been shown to give rise to discrete

135 populations of neurons (Flandin et al., 2010; Gelman et al., 2011, 2009). In order to fate 136 map BFCNs arising from either the MGE or POA, we crossed our ChAT $T^{F / p O}$ allele and 137 intersectional reporter with either $L h x 6^{\text {iCre }}$, marking the MGE, or Shh ${ }^{\text {Cre }}$, marking the POA, 138 and examined the labeled cells in P30 mice (Figure 2A).

140 These fate mapping experiments revealed that both MGE-derived (Lhx6 lineages) and 141 POA-derived (Shh lineages) include both deep- and superficially-projecting BFCNs, with 142 no statistically significant difference in layer projection density between the two 
143 populations (Figure 2D, E). The labeled BFCNs from both the MGE and POA had cell

144 bodies distributed throughout all nuclei of the basal forebrain, although we found that

145 the MGE-derived population gave rise to a slightly higher proportion of cells within the

146 medial septum (Bregma +0.5 to $+1.25, p=0.0018$ ) (Figure $2 \mathrm{~B}, \mathrm{C}$ ). Despite this, the two

147 populations had comparable projection densities across other cholinergic target regions,

148 including the hippocampus, a major target of the medial septum (Figure 2F). These

149 results led us to conclude that spatial origin is not a major source of cholinergic neuron

150 diversity. 
A

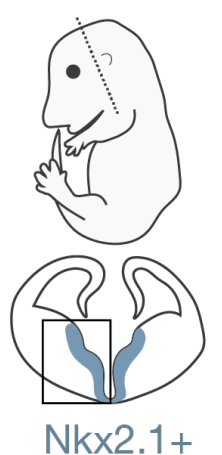

C

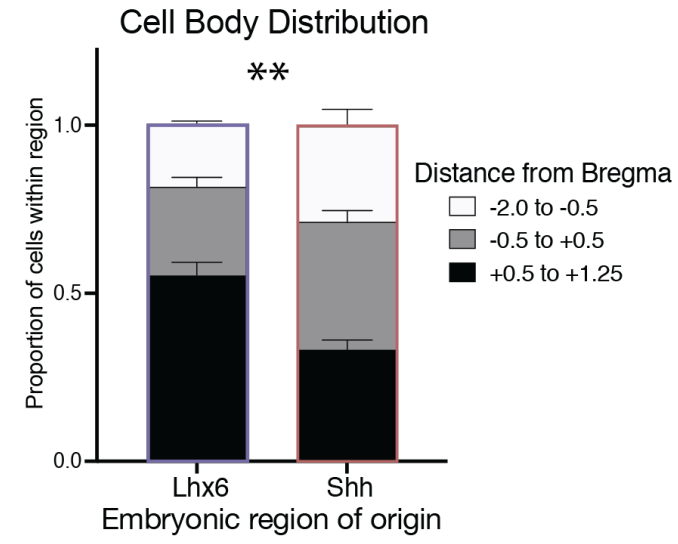

E

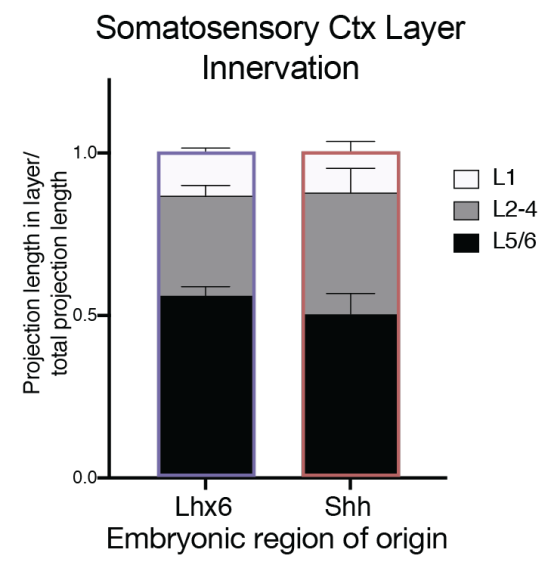

B
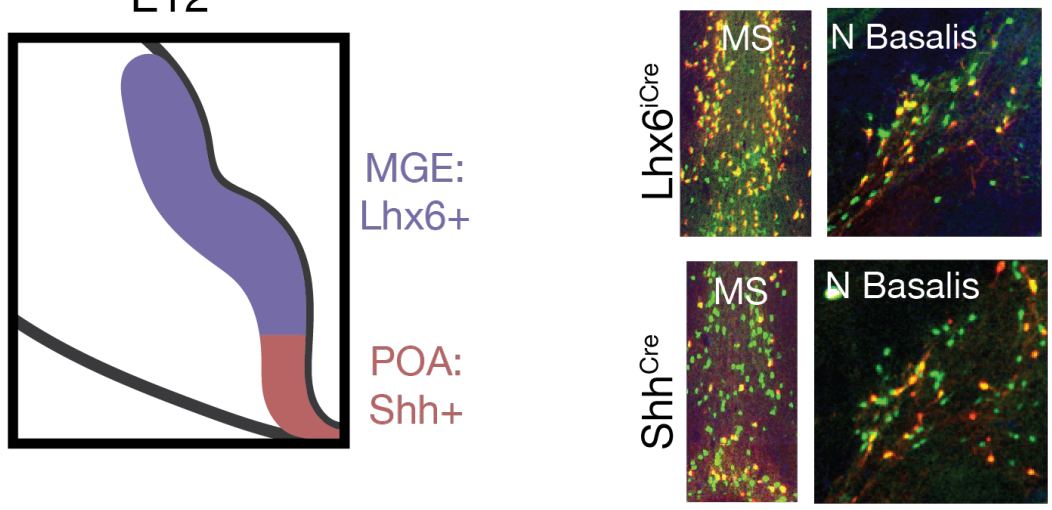

Ai65 ChAT

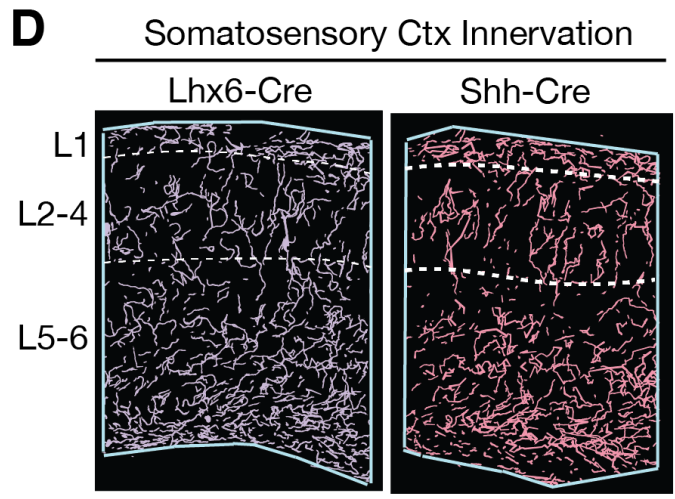

$\mathbf{F}$ Innervation in other regions

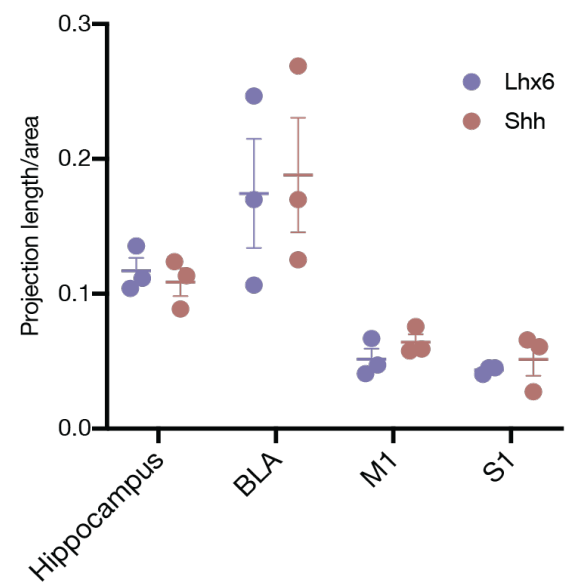

Figure 2. Spatial embryonic origin does not predict projection topography of cholinergic neurons. (A) Genetic strategy for fate mapping cells from the medial ganglionic eminence (MGE) or preoptic area (POA) based on differential gene expression. (B) Fate mapped cells in the P30 medial septum (MS) and nucleus basalis (N Basalis) from ChATFIpO;Lhx6iCre;Ai65 or ChATFipo;ShhCre;Ai65 animals stained with an anti-ChAT antibody. (C) Quantification of cell body distribution in the adult brain of cells originating in the MGE (Lhx6, $\mathrm{n}=3$ animals) or POA (Shh, $\mathrm{n}=4$ animals) (two-way ANOVA, $F(2,10)=9.25, p<0.0053$ ). (D) Reconstructions of axonal projections within a representative section of the P30 somatosensory cortex from fate-mapped brains. (E) Quantification of projection density within cortical layers for spatial fate mapped brains. Two-way ANOVA revealed no significant difference between groups $(F(2,6)=0.9034, p$ $=0.4311$ ) (Lhx6 $n=3$ animals, Shh $n=3$ animals). (F) Quantification of projection density within other cholinergic target regions revealed no significant differences between groups (two-way ANOVA, $F(2,6)=$ $0.1064, p=0.9552$ ). Each dot represents quantification for that region from an individual animal. 
152 Cellular birthdate predicts layer-specific cholinergic projection topography

154 An alternative source of neuronal diversity during development is cellular birthdate, i.e.,

155 the timing of a neuronal progenitor cell becoming terminally postmitotic. This principle 156 is evident in the ordering of pyramidal neurons (Angevine and Sidman, 1961) and

157 interneurons (Inan et al., 2012; Miyoshi et al., 2007) within cortical laminae. In order to

158 determine whether layer-specific cholinergic projections correspond with neuronal

159 birthdate, we again utilized the $C h A T^{F / p O}$ and Ai65 alleles, this time in combination with

160 an $A s c / 1^{C r e E R}$ driver (i.e., ChAT $\left.{ }^{F l p O} ; A s c / 1^{C r e E R} ; A i 65\right)$. Ascl1 is a proneural gene broadly but

161 transiently expressed in newly born neurons within the ventral embryonic telencephalon

162 (Casarosa et al., 1999). The expression trajectory of Asc/1 is closely tied to cellular 163 birthdate, in that its expression peaks as MGE-, septal, and POA-derived progenitors 164 exit the cell cycle, after which it is rapidly downregulated. In the context of the ChAT FlpO; 165 Asc/1 ${ }^{\text {CreER; }}$ Ai65 embryos, administration of tamoxifen at a given embryonic age allows 166 the birthdate of cholinergic cells to be captured (Battiste et al., 2007; Kim et al., 2008, 167 2011). Cholinergic cells exiting the cell cycle immediately following tamoxifen 168 administration (12-24 hour window) thereby express the tdTomato reporter 169 permanently.

171 In order to capture the timeframe during which most cholinergic neurons are generated,

172 we administered tamoxifen at E10, E11, E12, or E13, and then harvested the brains at 173 P30 to examine the axonal projection topography of labeled cholinergic neurons (Figure $1743 \mathrm{~A}, \mathrm{~B})$. Neurons projecting to the somatosensory cortex are generally born between E10- 
175 E12, while those labelled at E13 do not send projections to the somatosensory cortex

176 (or most other cortical regions) with the exception of the medial prefrontal cortex (see

177 below) (Figure 3D). Further analysis revealed that cholinergic neurons that become

178 postmitotic on different days have distinct projection patterns within the primary

179 somatosensory cortex, with early-born neurons projecting to deep layers and later born

180 neurons projecting progressively more superficially (ANOVA, $F(4,22)=48.20, p<$

181 0.0001). (Figure 3C, E). Axonal projections from E10-born cells were predominantly

182 located in L5-6, while those from E11-born cells were primarily in L2-4, and those from

183 E12-born cells were almost exclusively found in L1 of the primary somatosensory cortex.

184 These results indicate that the layer-specific cholinergic neurons that we previously

185 identified through viral injections become postmitotic at discrete embryonic ages. 

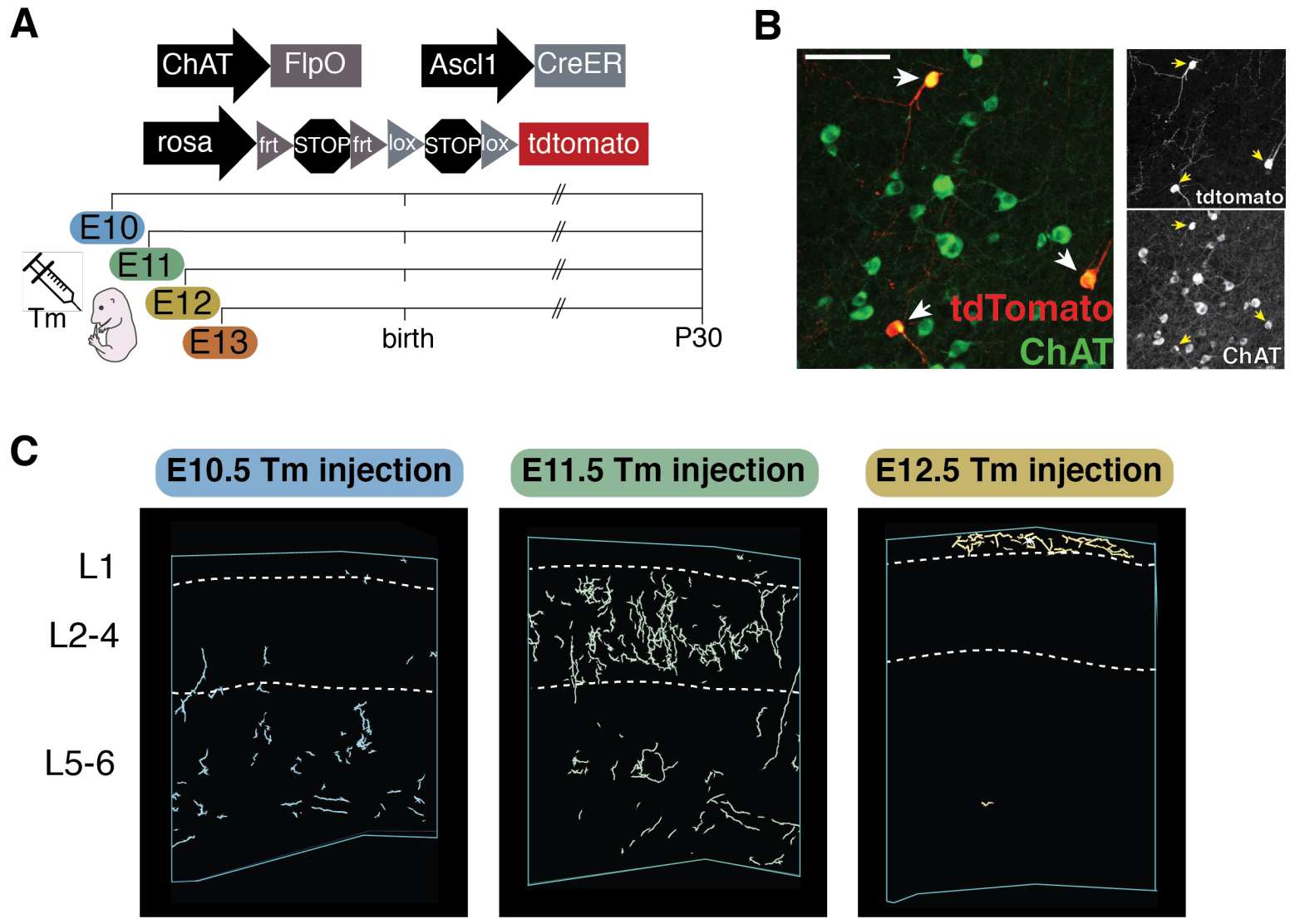

D Somatosensory Cortex Total Innervation

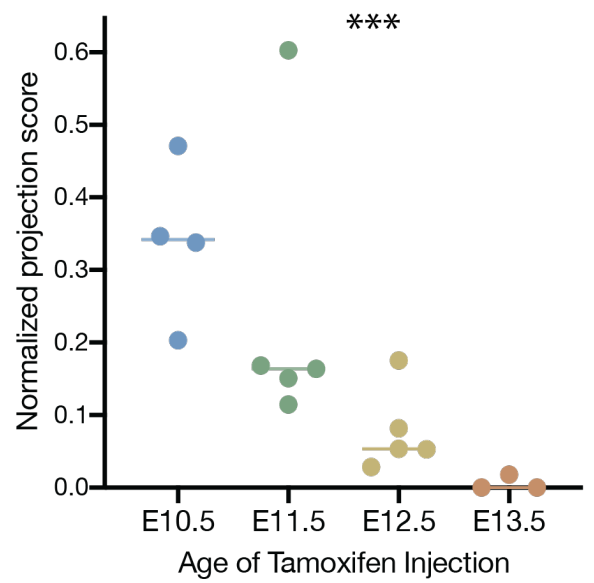

E Somatosensory Cortex Layer Innervation

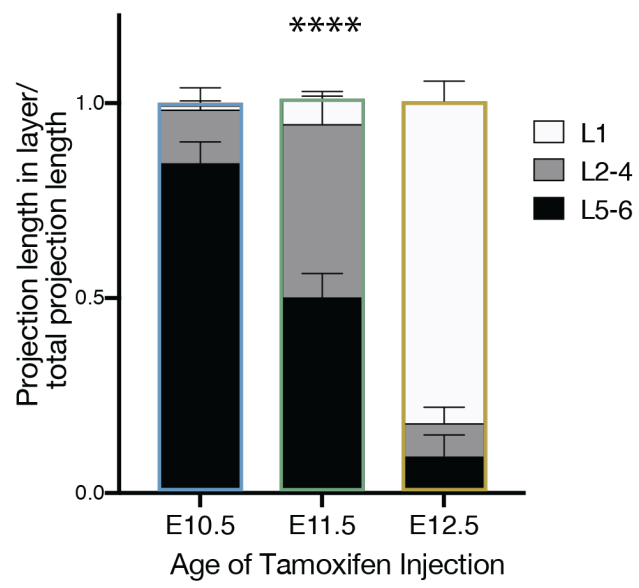

Figure 3. Cellular birthdate predicts layer-specific cholinergic projection topography in the primary somatosensory cortex. (A) Experimental design for tamoxifen (Tm) induction of CreER activity for neuronal birthdating. (B) Example of E11 tamoxifen-birthdated cholinergic neurons at P30 in the substantia innominate. Scale bar $=100 \mu \mathrm{m}$. (C) Reconstructions of birthdated cholinergic projections within representative sections of the P30 somatosensory cortex. E13 brains have little to no axonal projections within this region. (D) Quantification of overall somatosensory cortex innervation at P30 for cholinergic neurons born at each timepoint (One-way ANOVA $(F(3,13)=10.86, p=0.0008$ ). Each dot represents an individual animal. (E) Quantification of innervation to specific cortical layers for each birthdated cohort (Two-way ANOVA $(F(4,22)=48.20, p<0.0001)$. 
Birthdate predicts cholinergic projection topography throughout the forebrain

190 We also examined other cortical regions, including visual cortex (V1), primary motor

191 cortex (M1), and the medial prefrontal cortex (mPFC) in order to determine whether they

192 also contain layer-specific cholinergic projections. Importantly, we found that V1 shows

193 similar characteristics to S1. Cholinergic neurons born at later timepoints innervate

194 progressively more superficial layers of V1 and, like in S1, those born at E12

195 predominantly project to layer 1 (ANOVA, $F(4,24)=12.95, p<0.0001$, Figure 3 - figure

196 supplement $1 \mathrm{C}$ and D).

M1 and mPFC, conversely, show relatively weak layer-specificity overall, suggesting

201 L5/6, those born at E11 and E12 project across layers fairly indiscriminately (ANOVA (F(4, 202 14) $=2.514, p=0.0888)$, Figure 3 - figure supplement $1 \mathrm{~A}$ and $\mathrm{B})$. Likewise, in mPFC, 203 which is unique amongst cortical areas in receiving projections from E13 born cholinergic 204 neurons, early born cells primarily innervate deep layers, while later born cells appear to 205 target all layers (ANOVA $F(6,22)=5.678, p=0.0011$; Figure 4A, D, E).

207 Because the birthdating method employed here also captures cholinergic neurons 208 innervating other forebrain regions, we next examined the hippocampus and amygdala 209 to determine if cholinergic projections within these areas also correlated with cellular 210 birthdate. We found that hippocampally-projecting cholinergic neurons are primarily 
211 born at later timepoints (E12 and E13) (hippocampus - ANOVA, $F(3,14)=17.51, p<$

212 0.0001Figure 4B, F). Conversely, those projecting to the basolateral amygdala (BLA) are

213 primarily born at E11 and E12 (ANOVA, $F(3,11)=5.691, p=0.0133)$. Interestingly, BFCNs

214 born at E10 predominantly project to the central amygdala, which receives much less

215 overall cholinergic innervation than the BLA (ANOVA, $F(3,25)=308.5, p<0.0001)$ (Figure

$2164 \mathrm{C}, \mathrm{G}, \mathrm{H})$.

217

218 

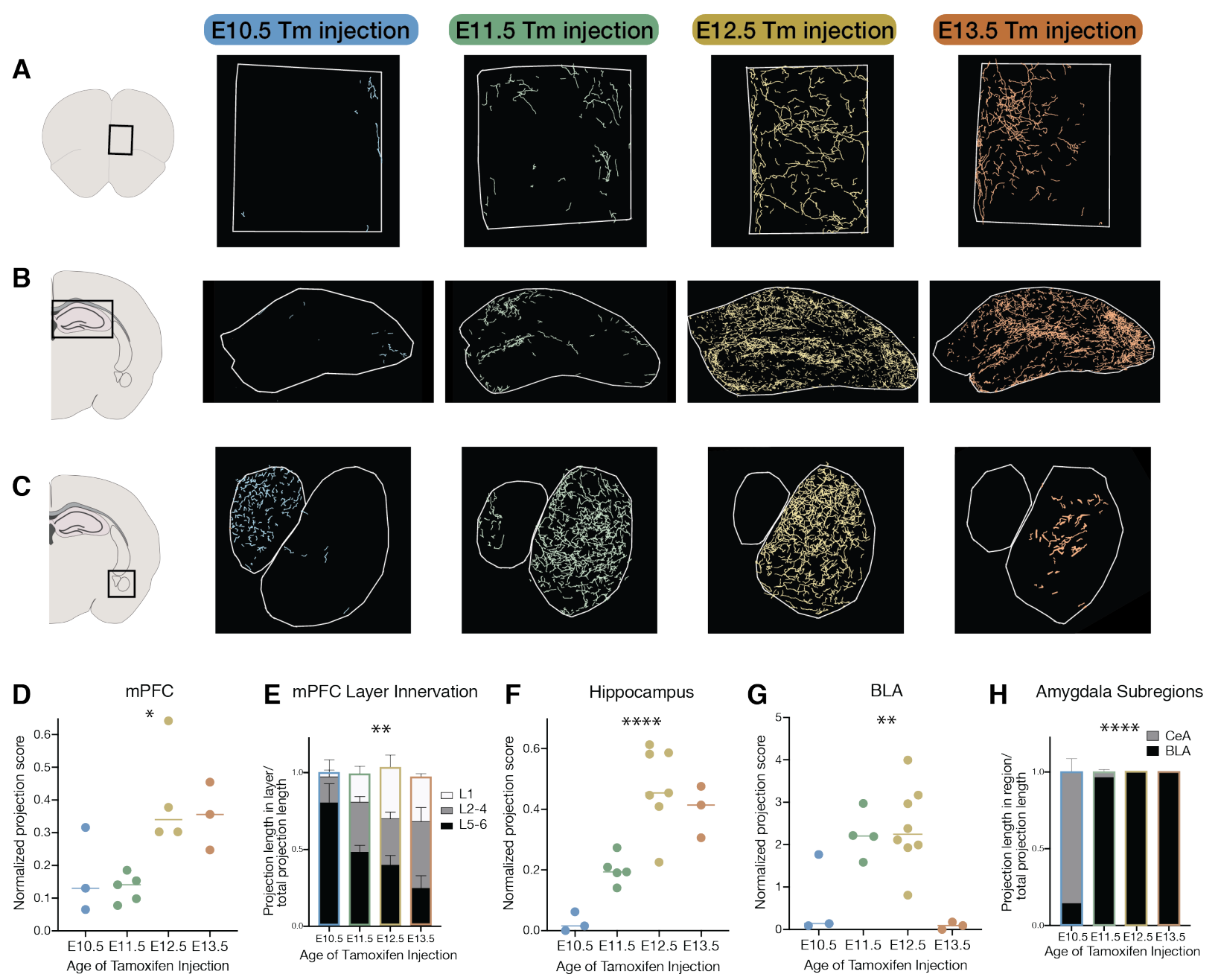

Figure 4. Birthdate predicts cholinergic topography in the hippocampus, amygdala, and mPFC. (A) Examples of birthdated cholinergic neuron projections to the medial prefrontal cortex (mPFC). (B) Examples of birthdated cholinergic neuron projections to the hippocampus at P30. (C) Examples of birthdated cholinergic neuron projections to the amygdala. Right, smaller outline represents central amygdala (CeA) and left, larger outline represents basolateral amygdala (BLA). (D) Quantification of total projections to the mPFC from birthdated cohorts of cholinergic neurons (one-way ANOVA, $F(3,11)=5.691$, $p=0.0133$ ). (E) Quantification of innervation to specific layers of MPFC for each birthdated cohort (Twoway ANOVA $(F(6,22)=5.678, p=0.0011)$. (F) Quantification of total projections to the hippocampus from birthdated cohorts of cholinergic neurons (one-way ANOVA, $F(3,14)=17.51, p<0.0001$ ). (G) Quantification of total projections to the amgydala from birthdated cohorts of cholinergic neurons (one-way ANOVA, $F(3,14)=8.219, p=0.0021)$. $(\mathrm{H})$ Quantification of projections to the CeA versus BLA for each birthdated timepoint (two-way ANOVA, $F(3,25)=308.5, p<0.0001$ ). Each dot in $D, E, F$, and $G$ represents an individual animal. 
220 Soma locations of birthdated cholinergic neurons

221

222 The cell bodies of cholinergic neurons born at each timepoint were found across the

223 rostral-caudal extent of the basal forebrain (Figure 5A, B). We noted a general trend that

224 early born (and deep-layer projecting) cells were biased towards the caudal-most

225 structures, while later born (and more superficially projecting) neurons were found more

226 rostrally. However, this difference was not statistically significant (ANOVA, $F(6,30)=$

227 2.103, $\mathrm{p}=0.0825)$. We repeated this experiment with EdU, an alternative birthdating

228 method that labels cell nuclei, and saw a similar trend that did reach statistical

229 significance (ANOVA, $F(6,24)=3.705, p=0.0092$; Figure $C, D)$. 

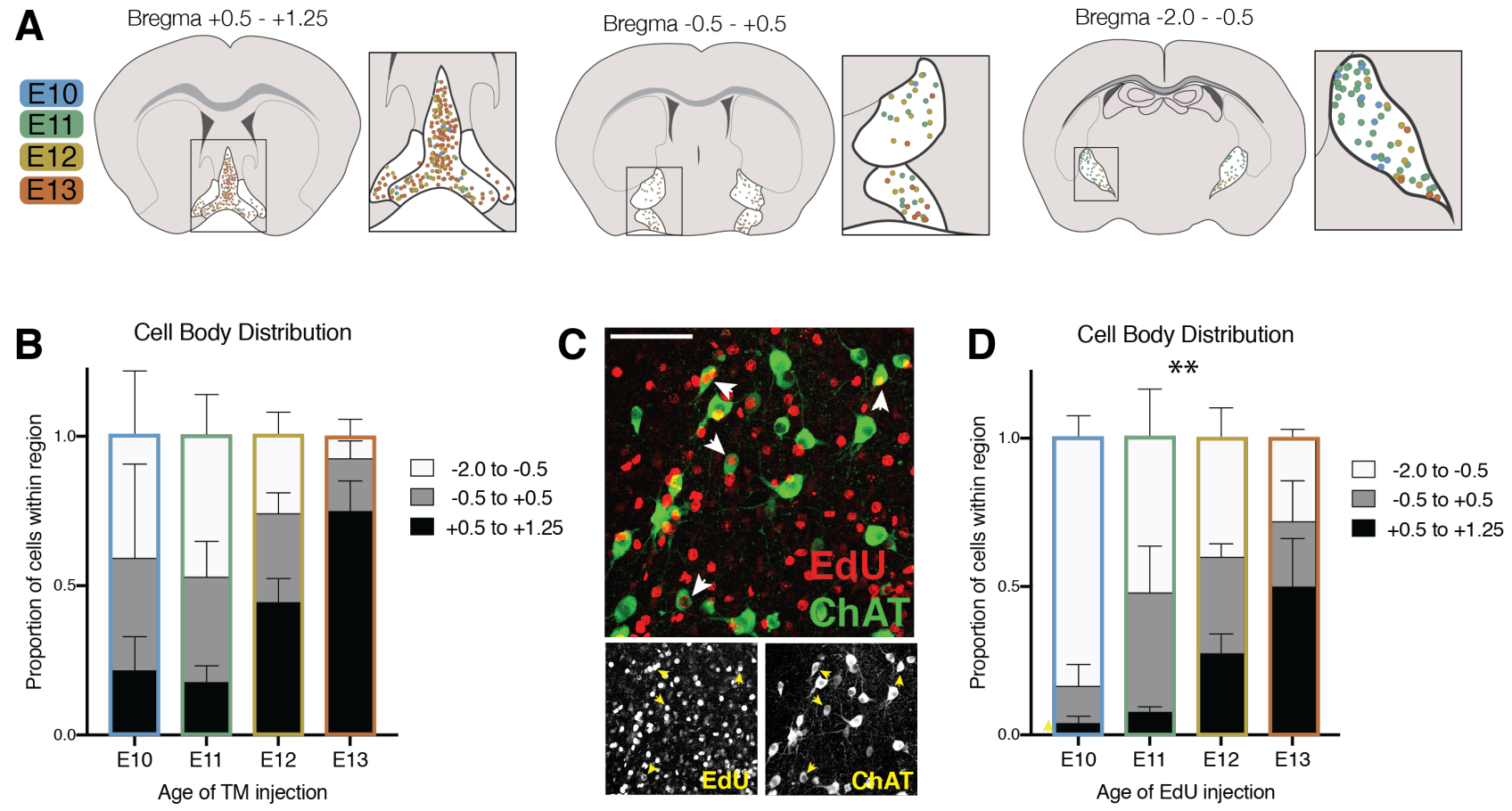

Figure 5. Cell body distribution of birthdated cholinergic neurons. (A) Cell body locations in the basal forebrain at P30 for cholinergic neurons born at each timepoint labeled by tamoxifen induction of CreER. (B) Quantification of cell body distribution (not significant by two-way ANOVA, $F(6,30)=2.103, p<0.0825$ ) $(E 10 n=3 ; E 11 n=5 ; E 12 n=8 ; E 13 n=3)$. (C) Birthdating using the alterative 5-Ethynyl-2'-deoxyuridine (EdU) method. Example image of P30 brain in which EdU was injected at E11, combining with a ChAT antibody stain to quantify cholinergic neurons. (D) Quantification of cell body distribution at P30 for cholinergic neurons birthdated with EdU (two-way ANOVA, $F(6,24)=3.705, p=0.0092)(\mathrm{n}=4$ for each timepoint). 
231 Cholinergic axons traverse distinct routes to their projection targets based on birthdate

233 Previous studies have described several routes that cholinergic axons take when

234 traversing the basal forebrain to reach the cortex. These axonal pathways have been

235 linked to projection target specificity (Bloem et al., 2014; Eckenstein et al., 1988; Saper,

236 1984). In order to determine whether cholinergic neurons born at different times

237 preferentially utilize specific axonal projection routes, we quantified the projections of

238 birthdated cholinergic neurons passing through the (1) rostromedial, (2) septal, (3)

239 rostrolateral, and (4) caudolateral pathways (Figure 6A). We found that E10 born

240 cholinergic neurons preferentially travel via the caudolateral pathway to reach deep

241 cortical layers (ANOVA, $(F(3,8=5.799, p=0.0209)$ (Figure 6B). Those born at E11, which

242 primarily project to layers $2-4$, were found to have most fibers in the rostrolateral and

243 septal pathways, suggesting these axons reach the cortex via either route (ANOVA,

$244 F(3,12)=20.14, p<0.0001) . \quad E 12$ and $\mathrm{E} 13$ born neurons, conversely, primarily travel

245 medially through the septal pathway (E12) (ANOVA, $F(3,8)=13.63, p=0.0016$ ) or the

246 rostro-medial pathway $(E 13)(A N O V A, F(3,8)=5.599, p=0.0230)$. Together, these results

247 suggest that cholinergic neurons projecting to deep layers of the somatosensory cortex

248 reach their targets by traversing through the lateral route, while the L1-specific

249 cholinergic neurons reach it via the septal pathway. E13 born neurons do not target most

250 of the cortex outside of the mPFC, and reach this target via the rostro-medial route.

252 To investigate this further, we again utilized the CAV-2::Cre retrograde virus, this time 253 injecting it directly into either the septal or caudolateral pathway of ChAT ${ }^{\text {FlpO; }}$ Ai65 mice. 
254 Quantification of cholinergic projections within the somatosensory cortex labeled using

255 this method confirmed that axons traveling via the septal route primarily arborize within

256 superficial layers, particularly layer 1 (Figure 6C, E). Conversely, those traveling via the

257 caudolateral route are relatively restricted to deep layers (Figure 6D, E). Together, these

258 results indicate that early born cholinergic neurons project their axons via the

259 caudolateral route to innervate deep layers of somatosensory cortex, while the axons of

260 later born cells traverse the septal route to innervate superficial layers. 
A

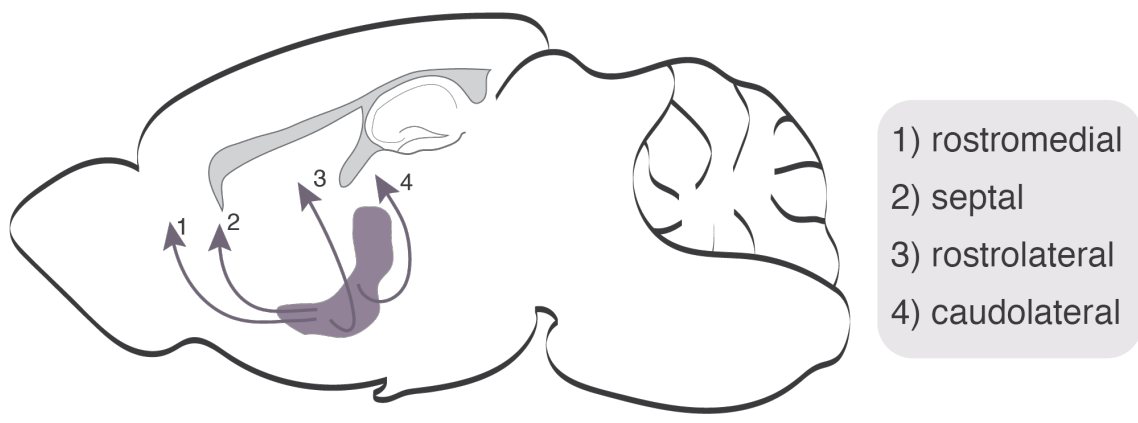

B E10.5 Tm injection

E11.5 Tm injection

E12.5 Tm injection

E13.5 Tm injection
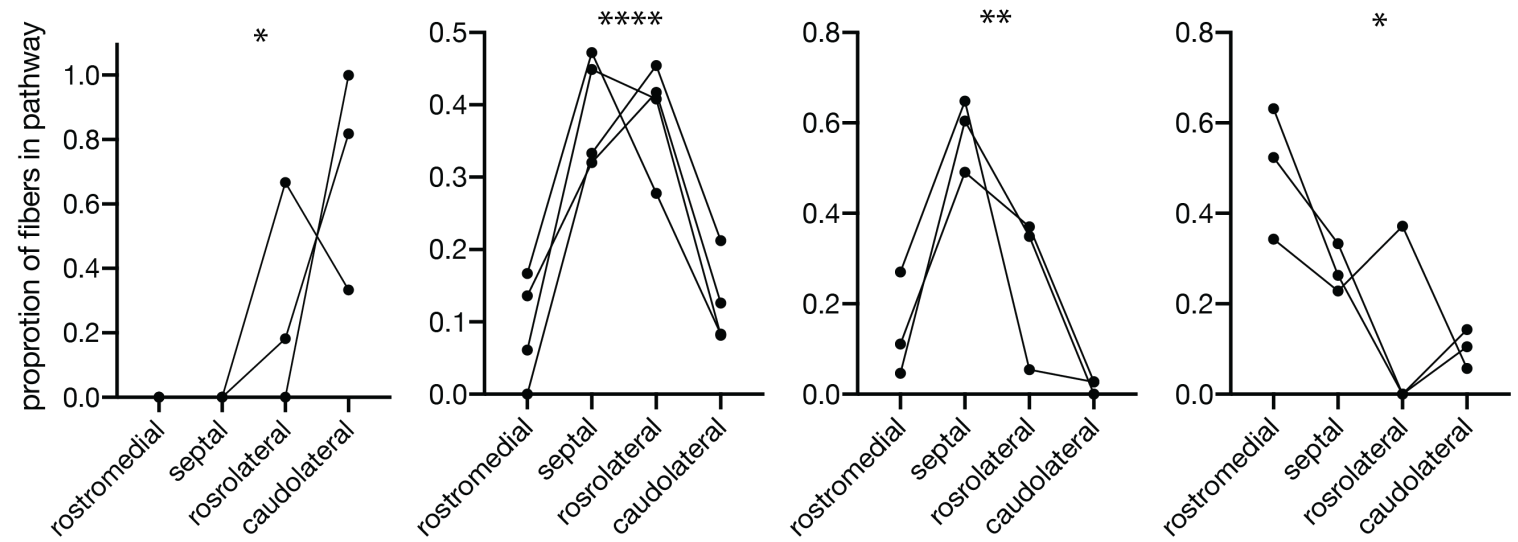

C

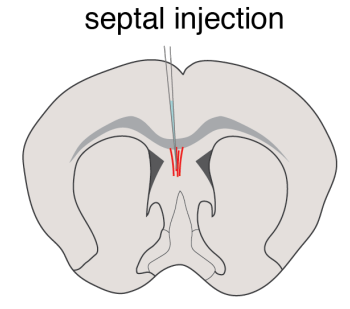

D caudolateral injection
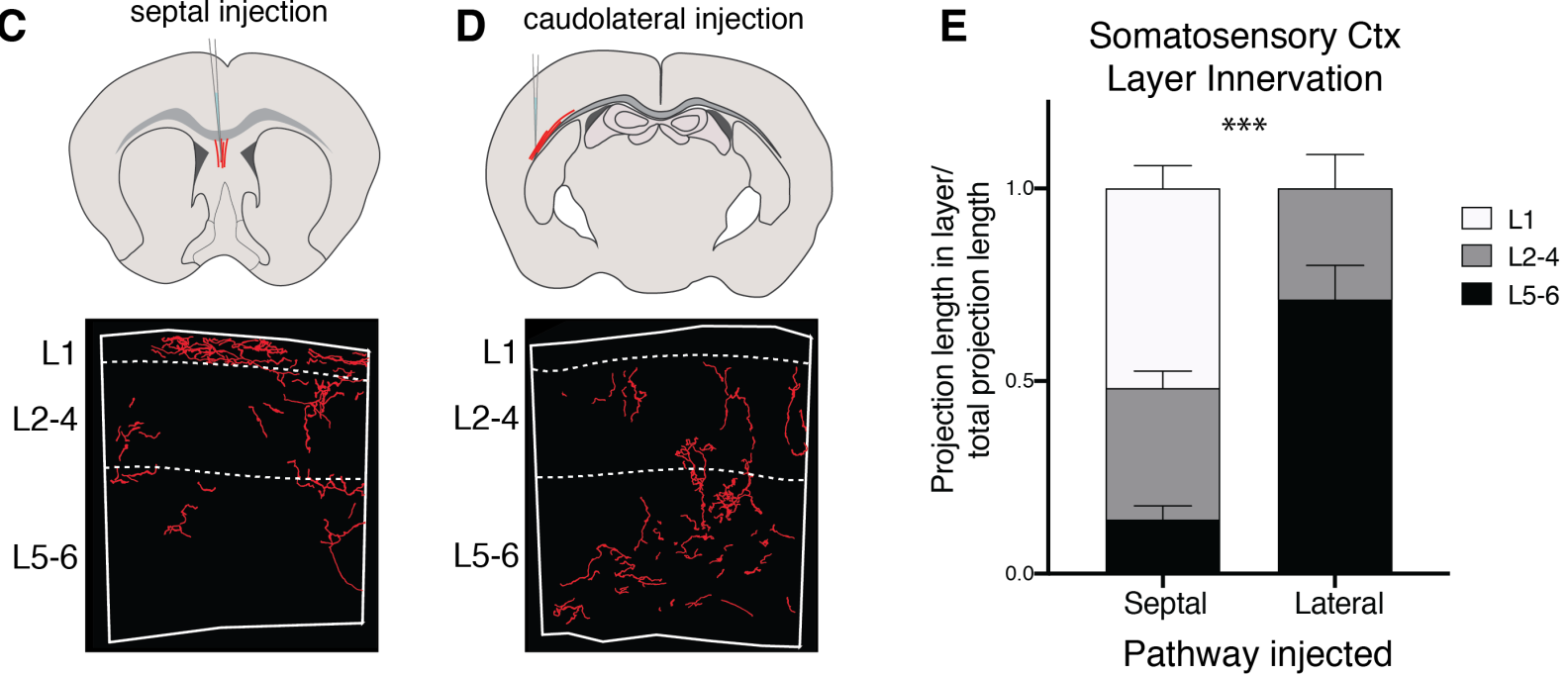

Figure 6. Cholinergic axons traverse distinct routes to their projection targets based on birthdate. (A) Routes cholinergic axons take to the cortex from the basal forebrain. (B) Proportion of fibers in each pathway for brains with birthdated cholinergic neurons at P30. Connected lines represent a single animal. One-way ANOVAs: $\mathrm{E} 10-F(3,8)=5.799, p=0.0209$; $\mathrm{E} 11-F(3,12)=20.14, p<0.0001 ; \mathrm{E} 12-F(3,8)=$ 13.63, $p=0.0016$; E13 $-F(3,8)=5.599, p=0.0230$. (C) Septal injection schematic (top) and example of fiber reconstruction in the somatosensory cortex (bottom). (D) Caudolateral injection schematic (top) and example of fiber reconstruction in the somatosensory cortex (bottom). (E) Quantification of innervation to specific layers in septal or caudolateral pathway injected brains ( $\mathrm{n}=3$ for each) (Two-way ANOVA, $F(2,8)$ $=28.87, p=0.0003$ ). 


\section{Discussion}

265 Although originally thought of as a diffuse, nonspecific source of neuromodulation in the

266 brain, BCFNs are now understood to function with great specificity and precision.

267 However, the organization of this system, how it develops, and how this organization

268 may mediate its specificity is still poorly understood. Here, we show that cholinergic

269 neurons targeting the somatosensory cortex innervate specific layers - an organizational

270 principle that was previously unknown and likely significantly contributes to the laminar

271 specificity of cholinergic signaling (Muñoz and Rudy, 2014; Muñoz et al., 2017;

272 Obermayer et al., 2017). Furthermore, we found that this specificity is determined by the

273 birthdate of cholinergic neurons in the BF, with early born cholinergic neurons primarily

274 targeting deep layers and later born neurons targeting progressively superficial layers of

275 the somatosensory cortex. Importantly, we identified a population of cholinergic neurons

276 whose axonal projections are almost completely restricted to layer 1. Cholinergic

277 neuronal birthdate is also correlated with projection topography in other target regions,

278 including other cortical areas, hippocampus, and amygdala. Finally, we found that

279 cholinergic neurons born at different times project via distinct axonal pathways to reach

280 their targets. Together, these findings extend our understanding of the organization of

281 BFCNs by revealing the relationship between their developmental origins and their

282 specific projection fields. 
286 Given the distinct actions of ACh in different cortical laminae, the existence of layer-

287 specific cholinergic neurons has been previously hypothesized. Here, using

288 intersectional genetics, we were able to identify layer-specific populations in the

289 somatosensory cortex for the first time. Perhaps most notably, we discovered the

290 existence of a population of cholinergic neurons, primarily born at E12 that almost

291 exclusively innervate layer 1 . This population may explain the high density of cholinergic

292 axons in L1 compared to other layers. Moreover, it has important functional implications

293 for ACh in cortical processes regulated by L1, such as cross-modal signaling.

295 Importantly, we observed marked differences in the degree of innervation specificity

296 within sensory (S1 and V1) and non-sensory (mPFC and M1) cortical regions. In a recent

297 anterograde tracing study, it was shown that labelled cohorts of cholinergic neurons

298 targeting the mPFC could be divided into two groups: one group projecting to all layers,

299 and another projecting to deep layers only (Bloem et al., 2014). Our results corroborate

300 this finding: projections from early born cholinergic neurons are relatively restricted to

301 deeper layers, while projections from later born cells were visible across all layers of

302 mPFC. M1 showed a similar trend, with some labeled cohorts of neurons showing deep

303 layer projection specificity, while others more diffusely innervated the entire cortical

304 mantle. This discrepancy between the degree of innervation specificity observed in

305 sensory versus non-sensory areas may relate to the distinct roles of cholinergic signaling

306 in information flow within these regions (bottom-up versus top-down, respectively). 
309 We initially expected that cholinergic neurons targeting different layers or regions might

310 originate from spatially distinct progenitor zones. Instead however we found no major

311 differences in the contribution of cholinergic cells that emanate from the MGE or POA

312 with regards to their cell body location or projection topography. Complementing our

313 results, a recent study fate mapped cholinergic neurons arising from the embryonic

314 septum, another Nkx2.1+ region. Like the MGE and POA, the septum gives rise to

315 cholinergic neurons whose axons have no apparent specificity within the cortex (Magno

316 et al., 2017).

318 By contrast, we discovered that cellular birthdate defines subpopulations of cholinergic

319 neurons with distinct projection distributions. How this specificity is achieved is unclear.

320 Are cholinergic populations specified as 'deep layer-targeting' or 'superficial layer-

321 targeting' as soon as they become postmitotic or do these reflect temporal shifts in

322 guidance cues that both dictate their projection trajectory and laminar specificity?

323 Although at present prohibitively challenging, one could potentially discern between

324 these possibilities through heterochronic transplantation of birthdated, postmitotic 325 cholinergic neurons.

327 Cholinergic neuron diversity

329 Our work adds a level of complexity in our understanding of cholinergic neuron diversity.

330 Cholinergic diversity likely extends beyond differences in their axonal targeting, with 331 subpopulations possessing distinct functional and electrophysiological properties. For 
332 example, a recently published study suggests that different basal forebrain cholinergic

333 cell types are responsible for the two timescales of ACh release in the cortex, with one

334 mediating slow, volume transmission and the other characterized by phasic, point-to-

335 point release (Laszlovszky et al., 2020). Additionally, it has recently been shown that

336 cholinergic neurons directly within the globus pallidus are more intimately involved in

337 basal ganglia circuitry and have specialized firing properties when compared to their

338 neighbors in the nucleus basalis (Saunders et al., 2015). In addition to the BFCNs, other

339 potential sources of ACh exist in the forebrain. For example, cholinergic neurons in the

340 pedunculopontine and laterodorsal tegmental nuclei of the brainstem innervate the basal

341 ganglia and thalamus, but do not appear to directly innervate most cortical areas

342 (Huerta-Ocampo et al., 2020; Martinez-Gonzalez et al., 2011). In addition, a population

343 of ChAT+, VIP+ interneurons are found within the cortex, although until recently it was

344 unclear whether these cells actually release ACh. Recent work, however, has shown that

345 they do appear to in some capacity release ACh (Granger et al., 2020; Obermayer et al.,

346 2019), although its functional role has yet to be fully resolved.

348 The recent advances in single-cell genomics technologies should provide useful insight

349 into the full diversity of forebrain cholinergic neurons. Linking molecular identities with

350 function and connectivity will be a crucial step towards understanding the diversity and

351 organization of this complex neuromodulatory system. 


\section{Methods}

355 Construction of the ChAT-ires-Flpo driver line

356 A targeting construct comprised of 5' and 3' homologous arms flanking an ires-Flpo-

357 polyA cassette and a floxed neo cassette for positive selection was electroporated into

358 C57BL/6 ES cells (B4). Correctly targeted ES cell clones were selected by long range

359 PCR and restriction mapping, and subsequently injected into recipient blastocysts to

360 create chimeric founder mice that were then bred with Cre deleter mice (CMV ${ }^{\text {Cre; Jax }}$

$361 \# 006054)$ to remove the neo cassette and obtain germline transmission. Once

362 established, heterozygous ChAT-ires-Flpo $\left(C h A T^{F / p O}\right)$ were bred with C57BL/6J mice (Jax

$363 \# 000664)$ to remove the Cre deleter allele, following which the ChAT ${ }^{F l p O}$ line was bred to

364 homozygosity.

365

366 Animals

367 All mouse colonies were maintained in accordance with the Institutional Animal Care and

368 Use Committees of NYU School of Medicine and Harvard Medical School. In addition to

369 ChAT $T^{F / p O}$ described above, the following mouse strains were used: Swiss Webster

370 (Taconic Biosciences), Asc/1 ${ }^{\text {CreER }}$ (Jax \#012882) (Kim et al., 2011), Lhx6 ${ }^{\text {iCre }}$ (Jax \#026555)

371 (Fogarty et al., 2007), Shh ${ }^{\text {eGFP-Cre }}$ (Jax \#005622) (Harfe et al., 2004), Ai65 (Jax \#021875)

372 (Madisen et al., 2015).

373

374 Cav-2::Cre injections 
375 Cav-2::Cre virus was obtained from the Plateforme de Vectorologie de Montpellier. Prior

376 to injections, mice were anesthetized using $5 \%$ isoflurane followed by maintenance on

$3772 \%$ isoflurane. To limit the spread of viral particles allowing for layer- or pathway-specific

378 injections, iontophoresis (Stoelting, digital Midgard precsion current source) was used

379 for all injections at 5 uA, 7 seconds on/3 seconds off, for a total of 10 minutes. The

380 following stereotaxic coordinates were used: deep layer S1 (AP: -1, ML: 3, DV: 0.85);

381 superficial layer S1 (AP: -1, ML: 3, DV: 0.15, 25º tilt), caudolateral pathway (AP: -1.4, ML:

382 4, DV: -2), medial pathway (AP: 1.7, ML: 0.25, DV: 3). All injections were performed on

383 mice aged P30-P33. 10 days following injection, mice were euthanized and perfused for

384 analysis.

385

386

Fate mapping spatial embryonic origins

$387 C h A T^{F / p O}$ mice were crossed with either $L h x 6^{i C r e}$ or Shh $h^{e G F P-C r e}$ mice to produce compound $388 C h A T^{F / p O} ; L h \times 6^{i C r e}$ and $C h A T^{F / p O} ; S h h^{\text {eGFP-Cre }}$ alleles. Males containing both $C h A T^{F / p O}$ and a 389 Cre allele were then crossed with female Ai65 mice and the resulting pups were 390 genotyped to identify individuals with all three alleles. At P30, these pups were 391 transcardially perfused with PBS and 4\% paraformaldehyde. Brains were harvested, 392 fixed overnight in $4 \%$ PFA at $4^{\circ} \mathrm{C}$, and sectioned at $50 \mu \mathrm{m}$ on a Leica VT $1200 \mathrm{~S}$ 393 Vibratome.

395 For projection and soma location quantification, sections were treated with a blocking 396 solution of $5 \%$ normal donkey serum, $0.3 \%$ Triton-X, and PBS for 30 minutes at room 397 temperature. Sections were then incubated in a primary antibody cocktail consisting of 
rabbit anti-DsRed (1:1000 dilution, Clontech 632496) and goat anti-ChAT (1:250 dilution, Millipore AB144P) overnight at $4^{\circ} \mathrm{C}$. After PBS washes, sections were incubated in secondary antibodies (Alexa Fluor 594 donkey anti-rabbit, Alexa Fluor 488 or 647 donkey anti-goat, 1:1000 dilution) in the dark for 1 hour at room temperature, followed by additional washes in PBS, treatment with DAPI, and mounting of sections on slides.

Birthdating with tamoxifen

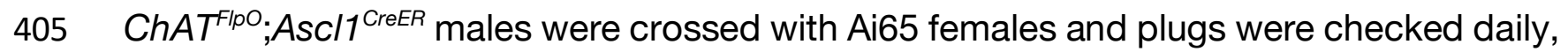
with the morning that a plug was observed being considered E0.5. Pregnant dams were 407 injected intraperitoneally with $2 \mathrm{mg}$ of tamoxifen (100 ul of $20 \mathrm{mg} / \mathrm{ml}$ tamoxifen, dissolved 408 in corn oil) between E10.5-E13.5. When pups were not delivered by noon on E19.5, pups were delivered by cesarian section and fostered. At P30, pups were transcardially 410 perfused with PBS and 4\% paraformaldehyde. Brains were harvested, fixed overnight in $4114 \%$ PFA at $4^{\circ} \mathrm{C}$, and sectioned at $50 \mu \mathrm{m}$ on a Leica VT $1200 \mathrm{~S}$ Vibratome.

413 For projection and soma location quantification, sections were treated with a blocking 414 solution of $5 \%$ normal donkey serum, $0.3 \%$ Triton-X, and PBS for 30 minutes at room 415 temperature. Sections were then incubated in a primary antibody cocktail consisting of 416 rabbit anti-DsRed (1:1000 dilution, Clontech 632496) and goat anti-ChAT (1:250 dilution, 417 Millipore AB144P) overnight at $4^{\circ} \mathrm{C}$. After PBS washes, sections were incubated in 418 secondary antibodies (Alexa Flour 594 donkey anti-rabbit, Alexa Flour 488 donkey anti419 goat, 1:1000 dilution) in the dark for 1 hour at room temperature, followed by additional 420 washes in PBS, treatment with DAPI, and mounting of sections on slides. 


\section{Birthdating with EdU}

423 Timed pregnant Swiss Webster females (Taconic) were injected with EdU (50 $\mu \mathrm{g}$ per $1 \mathrm{~g}$

424 body weight) between E10.5-E13.5. At P30, pups were transcardially perfused with PBS 425 and $4 \%$ paraformaldehyde. Brains were harvested, fixed overnight in $4 \% \operatorname{PFA}$ at $4^{\circ} \mathrm{C}$,

426 and sectioned at $50 \mu \mathrm{m}$ on a Leica VT $1200 \mathrm{~S}$ Vibratome. For soma location 427 quantification, sections were treated with a blocking solution of $5 \%$ normal donkey 428 serum, 0.3\% Triton-X, and PBS for 30 minutes at room temperature. Sections were then 429 incubated in goat anti-ChAT (1:250 dilution, Millipore AB144P) overnight at $4^{\circ} \mathrm{C}$. After 430 PBS washes, sections were incubated in Alexa Flour 594 donkey anti-goat (1:1000

431 dilution) in the dark for 1 hour at room temperature, followed by additional washes in 432 PBS. Sections were then treated with the Click-iT EdU kit for imaging Alexa Fluor 488 433 (ThermoFisher, C10337), followed by DAPI, additional PBS washes, and mounting of 434 sections on slides.

436 Soma location analysis

437 Temporally or spatially-fate mapped brains were sectioned and stained as described 438 above. Every eighth section from each brain was examined under a fluorescence 439 microscope and tdTomato+ cell bodies were annotated onto a brain atlas. For figures, 440 cell locations were approximated to their location on a representative atlas for that 441 rostro-caudal region. Cells were summed and the proportion of cell bodies within each 442 region per brain was calculated. ANOVA was used to determine whether significant 443 differences between the proportions of cells within each region existed across groups, 
444 with post-hoc tests using Sidak's (spatial fate mapping) or Tukey's (temporal fate 445 mapping) multiple comparisons tests.

447 Neurolucida reconstructions and projection quantification

448 For whole neuron reconstructions, brains were chosen with extremely sparse viral

449 labeling to ensure clarity assigning projections to the cell of interest. Sections containing

450 the cell of interest were imaged on a Zeiss LSM 800. Z-stacks of images were then

451 loaded into Neurolucida 360 (MBF Biosciences) and trees were reconstructed using the

452 'user guided' option with Directional Kernels. Contours of major features in the brain

453 sections were also traced for alignment of sections to produce the final reconstruction.

455 For the quantification of neuronal projections given brain regions, confocal images were

456 taken of relevant brain regions. The total projections within that region were

457 reconstructed in Neurolucida 360 and the total projection length was quantified using

458 Neurolucida explorer. To account for the fact that tamoxifen induction of CreER activity

459 results in a variable number of neurons labeled across brains, this quantification was

460 normalized in one of two ways. For overall projection density quantification within a given

461 region, total projection length was normalized to the number of cells labeled in that brain

462 to give a 'normalized projection score' (total projection length/number of cells $\left.{ }^{*} 1000\right)$. For

463 layer analysis, the projection within a given layer was simply quantified as a proportion

464 of the total projection length within that cortical region. 
467 Cholinergic axonal trajectories in birthdated brains were quantified by counting the

468 number of fiber segments within a given pathway (rostral, septal, rostrolateral, or

469 caudolateral). This number was then converted to a proportion relative to the

470 contribution of other pathways to account for variable labeling in cell number across

471 brains. Rostral pathway was considered as fibers running vertically (i.e., parallel to the

472 edge of the mPFC) in the superficial-most part of L1 of the medial cortex in sections

473 rostral to the medial septum. Fibers in the septal pathway were those in the medial part

474 of sections containing the septum that perforated the corpus callosum or ran vertically

475 through L1 in the cingulate cortex. The rostrolateral pathway was considered fibers

476 running through the external capsule in sections containing the medial septum or those

477 more rostral; the caudolateral pathway fibers were those in the external pathways in

478 sections caudal to this.

479

480 Sample Size

481 No statistical method was used to determine sample sizes. Sample size for each

482 experiment can be found in figure legends. For fate mapping experiments, mice from at 483 least two distinct litters per timepoint were used in analysis.

\section{Acknowledgements}

486 We thank the Fishell and Rudy laboratories for helpful feedback and discussion, G.

487 Pouchelon for critical reading of the manuscript, and N. Yusuf and M. Fernandez-Otero

488 for technical assistance. KCA is supported by NIH NRSA F31NS103398. BR is

489 supported by NIH P01NS074972, R01NS107257, and R01NS110079. RM is supported 
490 by NIH P01NS074972. GF is supported by NIH grants R01MH071679, R01NS081297,

491 5P01NS074972, and UG3MH120096, as well as support from the Simons Foundation

492 Award 566615. The ChAT-FlpO mouse was made in collaboration with the NYU Langone

493 Rodent Genetic Engineering Laboratory directed by Dr. Sang Yong Kim, with partial

494 support from P30CA016087.

495

496 Competing interests

497 The authors declare no competing interests.

498

499 Supplementary Files

500 Supplementary File 1: ANOVA summary tables and post-hoc test information.

501

502 Figure Supplements

503 [Figures and legends located after references]

504 Figure 1 - Figure Supplement 1. Precise and efficient recombination mediated by the

505 ChAT-iresFlpo driver

506 Figure 1 - Figure Supplement 2. Complete reconstruction of a L1-targeting cholinergic

507 projection neuron

508 Figure 1 - Figure Supplement 3. Complete reconstruction of a L5/6-targeting

509 cholinergic projection neuron.

510 Figure 3 - Figure Supplement 1. Birthdated cholinergic neuron projections to the

511 primary motor and primary visual cortices. 


\section{References}

Angevine, J.B., and Sidman, R.L. (1961). Autoradiographic study of cell migration during histogenesis of cerebral cortex in the mouse. Nature 192, 766-768.

Ballinger, E.C., Ananth, M., Talmage, D.A., and Role, L.W. (2016). Basal Forebrain Cholinergic Circuits and Signaling in Cognition and Cognitive Decline. Neuron 91, 518 1199-1218.

Bandler, R.C., Mayer, C., and Fishell, G. (2017). Cortical interneuron specification: the juncture of genes, time and geometry. Curr. Opin. Neurobiol. 42, 17-24.

Baskerville, K.A., Chang, H.T., and Herron, P. (1993). Topography of cholinergic afferents from the nucleus basalis of meynert to representational areas of sensorimotor cortices in the rat. J. Comp. Neurol. 335, 552-562.

524 Battiste, J., Helms, A.W., Kim, E.J., Savage, T.K., Lagace, D.C., Mandyam, C.D., Eisch, A.J., Miyoshi, G., and Johnson, J.E. (2007). Ascl 1 defines sequentially generated lineage-resricted neuronal and oligodendrocyte precursor cells in the spinal cord. Development 134, 285-293.

Bloem, B., Schoppink, L., Rotaru, D.C., Faiz, A., Hendriks, P., Mansvelder, H.D., van de Berg, W.D.J., and Wouterlood, F.G. (2014). Topographic mapping between basal forebrain cholinergic neurons and the medial prefrontal cortex in mice. J. Neurosci. 34, 531 16234-16246.

Casarosa, S., Fode, C., and Guillemot, F. (1999). Mash1 regulates neurogenesis in the ventral telencephalon. Development 126, 525-534.

534 Eckenstein, F.P., Baughman, R.W., and Quinn, J. (1988). Anatomical Study of 535 Cholinergic Cortex. Sci. York 25, 457-474.

Ekstrand, M.I., Nectow, A.R., Knight, Z.A., Latcha, K.N., Pomeranz, L.E., and Friedman, J.M. (2014). Molecular profiling of neurons based on connectivity. Cell 157, 1230-1242.

538 Flames, N., Pla, R., Gelman, D.M., Rubenstein, J.L.R., Puelles, L., and Marín, O. (2007). Delineation of multiple subpallial progenitor domains by the combinatorial expression of transcriptional codes. J. Neurosci. 27, 9682-9695.

541 Flandin, P., Kimura, S., and Rubenstein, J.L.R. (2010). The progenitor zone of the 542 ventral medial ganglionic eminence requires Nkx2-1 to generate most of the globus 543 pallidus but few neocortical interneurons. J. Neurosci. 30, 2812-2823.

544 Fogarty, M., Grist, M., Gelman, D., Marín, O., Pachnis, V., and Kessaris, N. (2007). 545 Spatial genetic patterning of the embryonic neuroepithelium generates GABAergic 546 interneuron diversity in the adult cortex. J. Neurosci. 27, 10935-10946. 
547 Froemke, R.C., Merzenich, M.M., and Schreiner, C.E. (2007). A synaptic memory trace 548 for cortical receptive field plasticity. Nature 450, 425-429.

549 Gelman, D., Griveau, A., Dehorter, N., Teissier, A., Varela, C., Pla, R., Pierani, A., and 550 Marin, O. (2011). A wide diversity of cortical GABAergic interneurons derives from the 551 embryonic preoptic area. J Neurosci 31, 16570-16580.

552 Gelman, D.M., Martini, F.J., Nóbrega-Pereira, S., Pierani, A., Kessaris, N., and Marín, 553 O. (2009). The embryonic preoptic area is a novel source of cortical GABAergic 554 interneurons. J. Neurosci. 29, 9380-9389.

555 Granger, A.J., Wang, W., Robertson, K., El-Rifai, M., Zanello, A.F., Bistrong, K., Saunders, A., Chow, B.W., Nuñez, V., Turrero García, M., et al. (2020). Cortical ChAT+ neurons co-transmit acetylcholine and GABA in a target- and brain-region-specific manner. Elife 9, 264-277.

Hansen, D. V, Lui, J.H., Flandin, P., Yoshikawa, K., Rubenstein, J.L., Alvarez-Buylla, A., and Kriegstein, A.R. (2013). Non-epithelial stem cells and cortical interneuron

Harfe, B.D., Scherz, P.J., Nissim, S., Tian, H., Mcmahon, A.P., and Tabin, C.J. (2004). Evidence for an Expansion-Based Temporal Shh Gradient in Specifying Vertebrate Digit Identities protein (Driever and Nusslein-Volhard, 1988) to the pat-terning of the ventral neural tube by different concentra-tions of Sonic hedgehog (reviewed in 566 McMahon. Cell 118, 517-528.

567 Huerta-Ocampo, I., Hacioglu-Bay, H., Dautan, D., and Mena-Segovia, J. (2020).

568 Distribution of midbrain cholinergic axons in the thalamus. ENeuro 7, 1-10.

569 Inan, M., Welagen, J., and Anderson, S.A. (2012). Spatial and temporal bias in the 570 mitotic origins of somatostatin- and parvalbumin-expressing interneuron subgroups 571 and the chandelier subtype in the medial ganglionic eminence. Cereb. Cortex 22, 820572827.

573 Jensen, P., Farago, A.F., Awatramani, R.B., Scott, M.M., Deneris, E.S., and Dymecki, 574 S.M. (2008). Redefining the serotonergic system by genetic lineage. Nat. Neurosci. 11, 575 417-419.

576 Junyent, F., and Kremer, E.J. (2015). CAV-2 - Why a canine virus is a neurobiologist's 577 best friend. Curr. Opin. Pharmacol. 24, 86-93.

578 Kim, E.J., Battiste, J., Nakagawa, Y., and Johnson, J.E. (2008). Ascl1 (Mash1) lineage 579 cells contribute to discrete cell populations in CNS architecture. Mol. Cell. Neurosci. $58038,595-606$.

581 Kim, E.J., Ables, J.L., Dickel, L.K., Eisch, A.J., and Johnson, J.E. (2011). Ascl1 (Mash1) 
582

583

584

585

586

587

588

589

590

591

592

593

594

595

596

597

598

599

600

601

602

603

604

605

606

607

608

609

610

611

612

613

614

615

616

defines cells with long-term neurogenic potential in subgranular and subventricular zones in adult mouse brain. PLoS One 6.

Laszlovszky, T., Schlingloff, D., Hegedüs, P., Freund, T.F., Gulyás, A., Kepecs, A., and Hangya, B. (2020). Distinct synchronization, cortical coupling and behavioral function of two basal forebrain cholinergic neuron types. Nat. Neurosci. 703090.

Li, X., Yu, B., Sun, Q., Zhang, Y., Ren, M., Zhang, X., Li, A., Yuan, J., Madisen, L., Luo, Q., et al. (2018). Generation of a whole-brain atlas for the cholinergic system and mesoscopic projectome analysis of basal forebrain cholinergic neurons. Proc. Natl. Acad. Sci. 115, 415-420.

Lim, L., Mi, D., Llorca, A., and Marín, O. (2018). Development and Functional Diversification of Cortical Interneurons. Neuron.

Luchicchi, A., Bloem, B., Viaña, J.N.M., Mansvelder, H.D., and Role, L.W. (2014). Illuminating the role of cholinergic signaling in circuits of attention and emotionally salient behaviors. Front. Synaptic Neurosci. 6, 24.

Madisen, L., Garner, A.R., Shimaoka, D., Chuong, A.S., Klapoetke, N.C., Li, L., van der Bourg, A., Niino, Y., Egolf, L., Monetti, C., et al. (2015). Transgenic mice for intersectional targeting of neural sensors and effectors with high specificity and performance. Neuron 85, 942-958.

Magno, L., Barry, C., Schmidt-Hieber, C., Theodotou, P., Häusser, M., and Kessaris, N. (2017). NKX2-1 Is Required in the Embryonic Septum for Cholinergic System Development, Learning, and Memory. Cell Rep. 20, 1572-1584.

Marin, O., Anderson, S.A., and Rubenstein, J.L. (2000). Origin and molecular specification of striatal interneurons. J. Neurosci. 20, 6063-6076.

Martinez-Gonzalez, C., Bolam, J.P., and Mena-Segovia, J. (2011). Topographical Organization of the Pedunculopontine Nucleus. Front. Neuroanat. 5, 1-10.

Mesulam, M.M., Mufson, E.J., Wainer, B.H., and Levey, A.I. (1983). Central cholinergic pathways in the rat: an overview based on an alternative nomenclature (Ch1-Ch6). Neuroscience 10, 1185-1201.

Miyoshi, G., Butt, S.J.B., Takebayashi, H., and Fishell, G. (2007). Physiologically distinct temporal cohorts of cortical interneurons arise from telencephalic Olig2expressing precursors. J. Neurosci. 27, 7786-7798.

Muñoz, W., and Rudy, B. (2014). Spatiotemporal specificity in cholinergic control of neocortical function. Curr. Opin. Neurobiol. 26, 149-160.

Muñoz, W., Tremblay, R., Levenstein, D., and Rudy, B. (2017). Layer-specific modulation of neocortical dendritic inhibition during active wakefulness. Science (80-. ). 
355, 954-959.

618 Nóbrega-Pereira, S., Gelman, D., Bartolini, G., Pla, R., Pierani, A., and Marín, O. (2010). Origin and Molecular Specification of Globus Pallidus Neurons. J. Neurosci. 30, 28246202834.

621 Obermayer, J., Verhoog, M.B., Luchicchi, A., and Mansvelder, H.D. (2017). Cholinergic 622 modulation of cortical microcircuits is layer-specific: Evidence from rodent, monkey 623 and human brain. 11, 1-12.

624 Obermayer, J., Luchicchi, A., Heistek, T.S., de Kloet, S.F., Terra, H., Bruinsma, B., 625 Mnie-Filali, O., Kortleven, C., Galakhova, A.A., Khalil, A.J., et al. (2019). Prefrontal 626 cortical ChAT-VIP interneurons provide local excitation by cholinergic synaptic 627 transmission and control attention. Nat. Commun. 10,1-14.

628 Patel, J.C., Rossignol, E., Rice, M.E., and Machold, R.P. (2012). Opposing regulation of 629 dopaminergic activity and exploratory motor behavior by forebrain and brainstem cholinergic circuits. Nat. Commun. 3, 1172.

631 Picciotto, M.R., Higley, M.J., and Mineur, Y.S. (2012). Acetylcholine as a 632 neuromodulator: cholinergic signaling shapes nervous system function and behavior. 633 Neuron 76, 116-129.

634 Robertson, S.D., Plummer, N.W., de Marchena, J., and Jensen, P. (2013).

635 Developmental origins of central norepinephrine neuron diversity. Nat. Neurosci. 16, 636 1016-1023.

637 Robertson, S.D., Plummer, N.W., and Jensen, P. (2016). Uncovering diversity in the 638 development of central noradrenergic neurons and their efferents. Brain Res. 1641, 639 234-244.

640 Saper, C.B. (1984). Organization of cerebral cortical afferent systems in the rat. II. 641 Magnocellular basal nucleus. J. Comp. Neurol. 222, 313-342.

642 Sarter, M., Parikh, V., and Howe, W.M. (2009). Phasic acetylcholine release and the 643 volume transmission hypothesis: Time to move on. Nat. Rev. Neurosci. 10, 383-390.

644 Saunders, A., Oldenburg, I. a, Berezovskii, V.K., Johnson, C. a, Kingery, N.D., Elliott, 645 H.L., Xie, T., Gerfen, C.R., and Sabatini, B.L. (2015). A direct GABAergic output from 646 the basal ganglia to frontal cortex. Nature 05, [In Press].

647 Wonders, C.P., Taylor, L., Welagen, J., Mbata, I.C., Xiang, J.Z., and Anderson, S. a. 648 (2008). A spatial bias for the origins of interneuron subgroups within the medial 649 ganglionic eminence. Dev. Biol. 314, 127-136.

650 Wu, H., Williams, J., and Nathans, J. (2014). Complete morphologies of basal forebrain 651 cholinergic neurons in the mouse. Elife 3, 1-17. 
652 Xu, Q., Tam, M., and Anderson, S.A. (2008). Fate mapping Nkx2.1-lineage cells in the 653 mouse telencephalon. J. Comp. Neurol. 506, 16-29.

654 Zaborszky, L., Csordas, A., Mosca, K., Kim, J., Gielow, M.R., Vadasz, C., and 655 Nadasdy, Z. (2015). Neurons in the basal forebrain project to the cortex in a complex 656 topographic organization that reflects corticocortical connectivity patterns: An 657 experimental study based on retrograde tracing and 3D reconstruction. Cereb. Cortex 658 25, 118-137. 

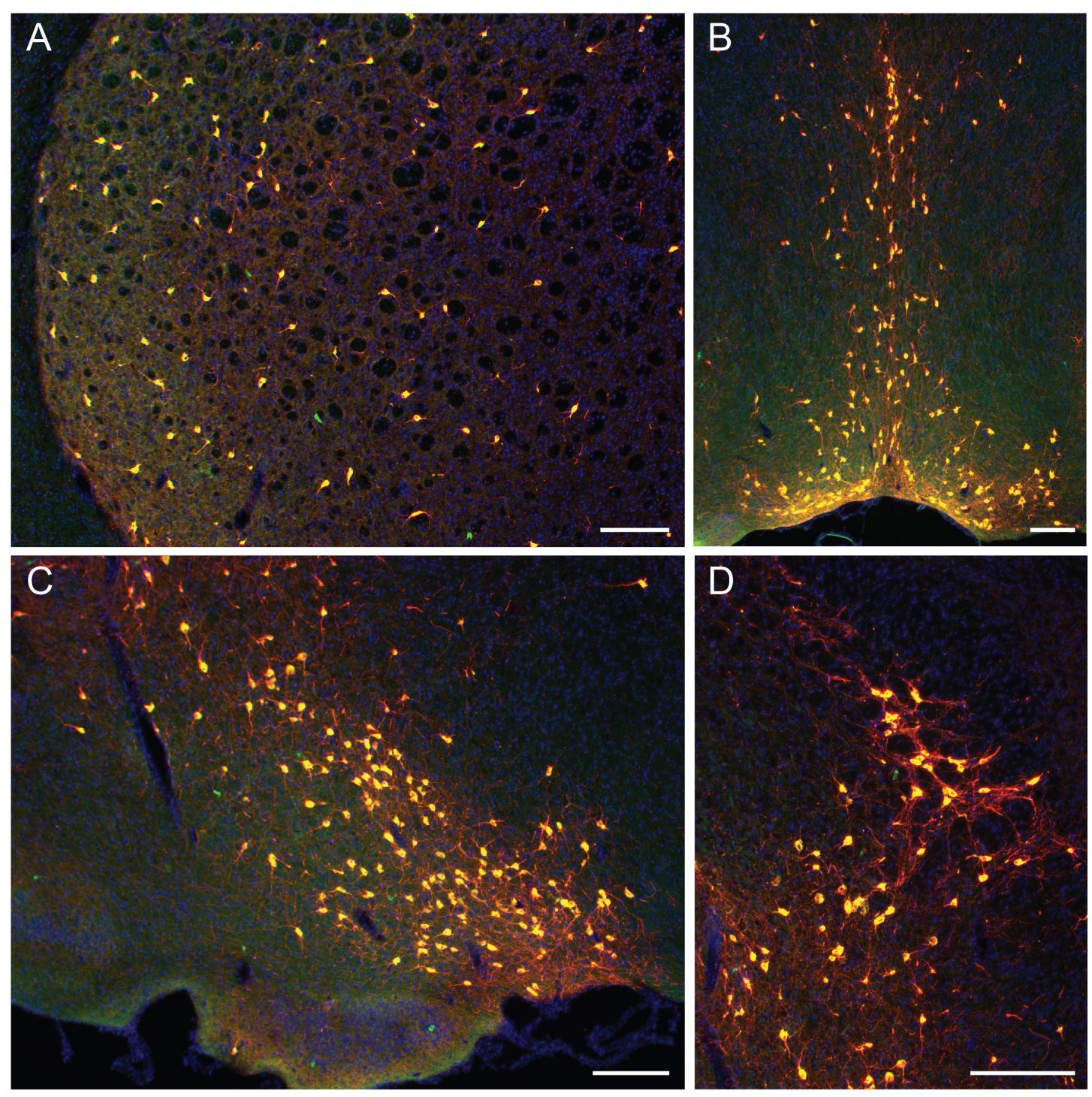

Figure 1 - Figure Supplement 1. Precise and efficient recombination mediated by the ChATiresFIpo driver. (A-D) ChAT immunohistochemstiry (IHC; green) performed on coronal tissue sections (20 $\mu \mathrm{m}$ thich) from P17 ChATFlpo; Dlx6aCre (pan-ventral forebrain driver); Ai65 brains. ChAT+ cells labeled by the ChAT-resFlpo driver express tdTomato (red) in this cross. (A) striatum: 95\% of ChAT neurons identified by ChAT IHC xo-express tdTomato (527/555 cells counted). (B) medial septum/diagonal band: $99 \% \quad(576 / 580$ cells counted). (C-D) diagonal band/substantia innominata/nucleus basalis: $97 \%$ (437/449 cells counted). Scale bars in all panels represent $300 \mu \mathrm{m}$. 


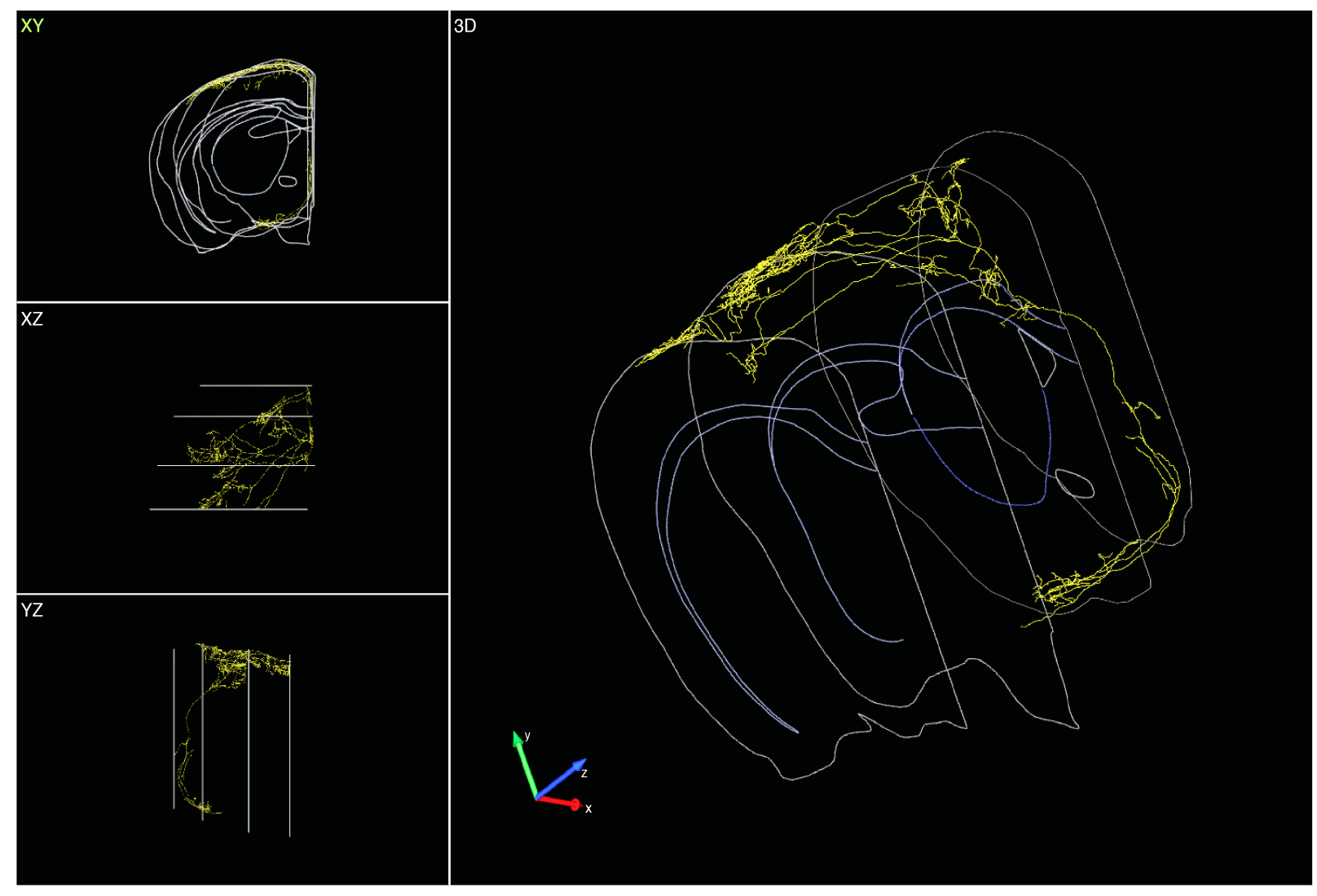

Figure 1 - Figure Supplement 2. Complete reconstruction of a L1-targeting cholinergic projection neuron. Left top, front view. Left middle, top view. Left bottom, side view. Right, 3D view. 


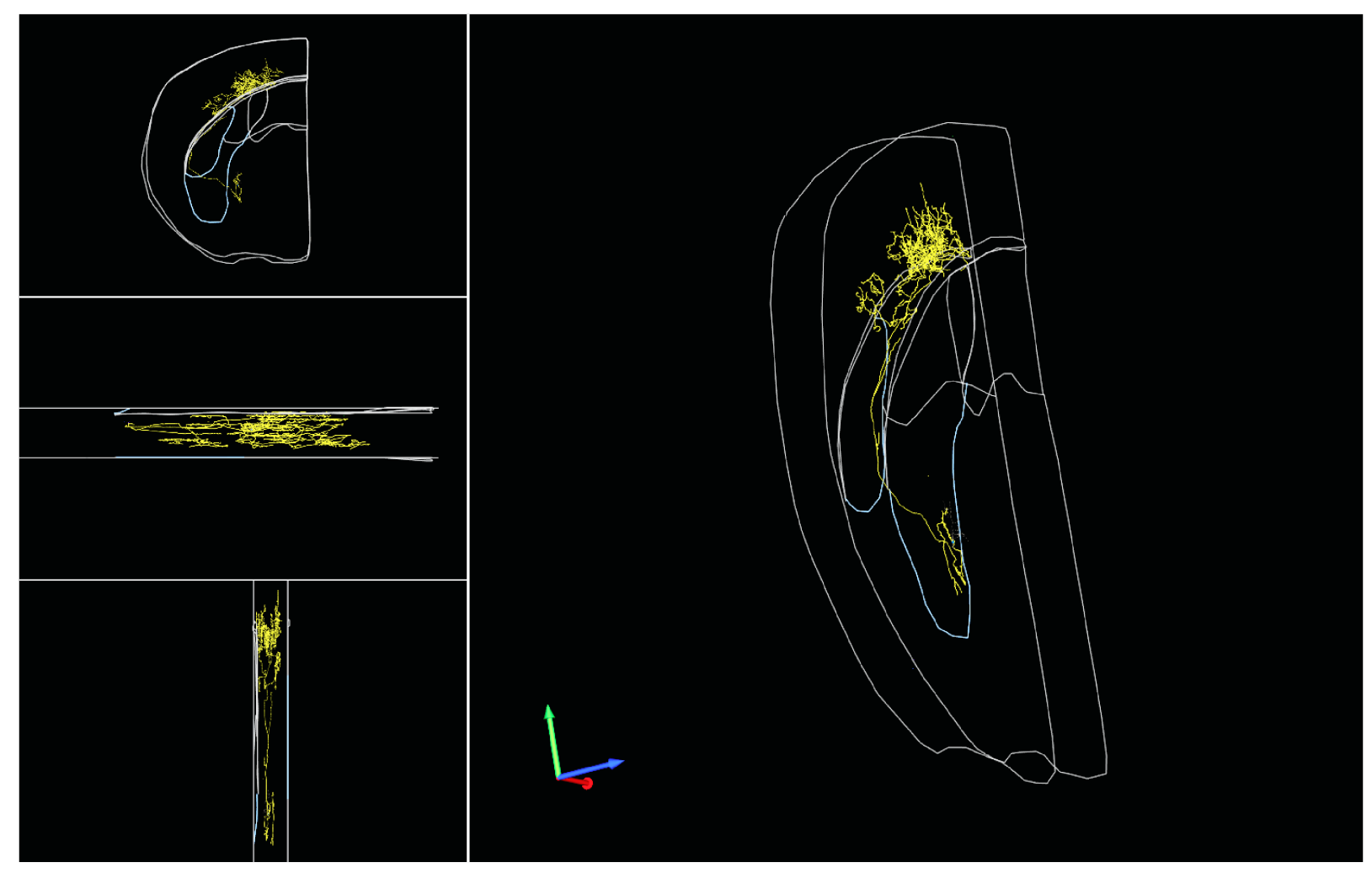

Figure 1 - Figure Supplement 3. Complete reconstruction of a L5/6-targeting cholinergic projection neuron. Left top, front view. Left middle, top view. Left bottom, side view. Right, 3D view. 


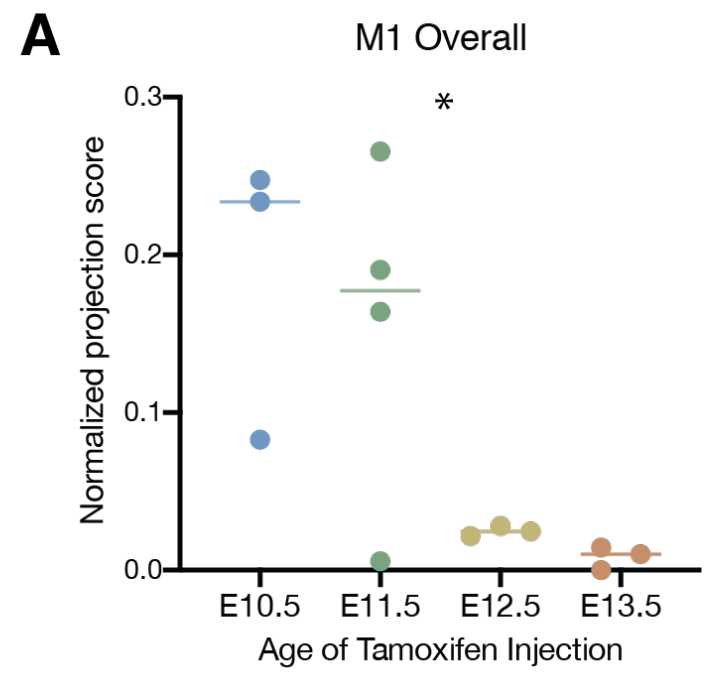

B

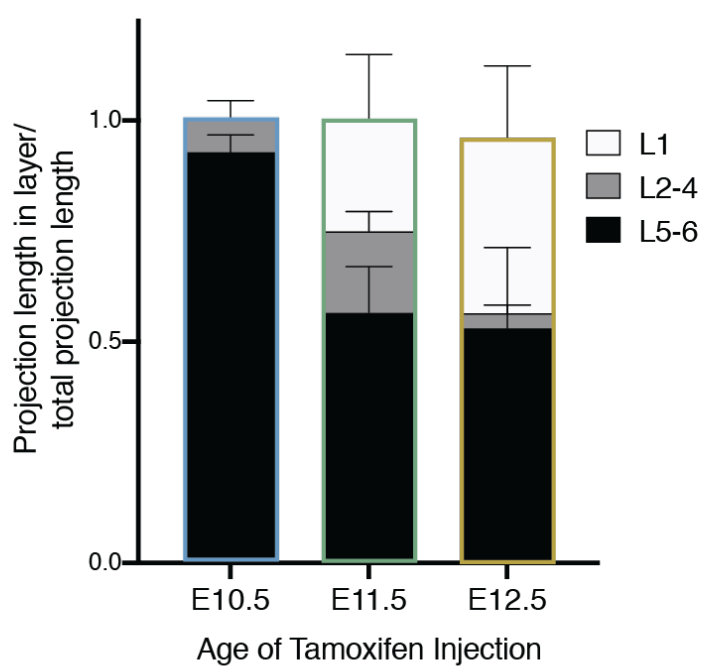

C V1 Overall

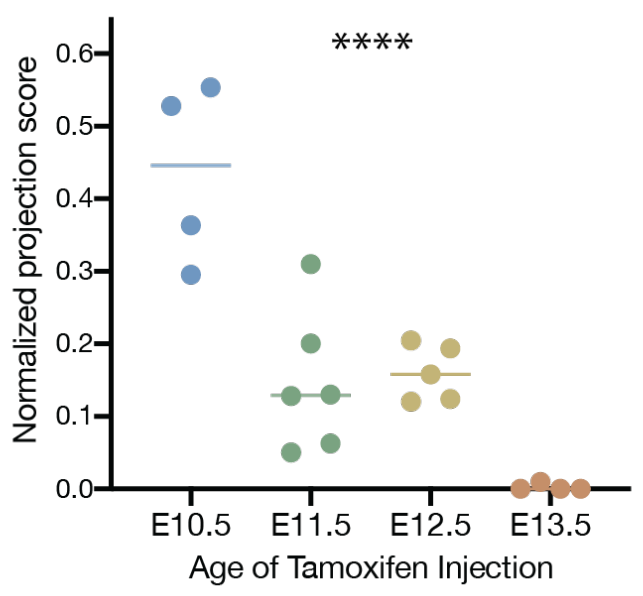

D V1 Layer Innervation

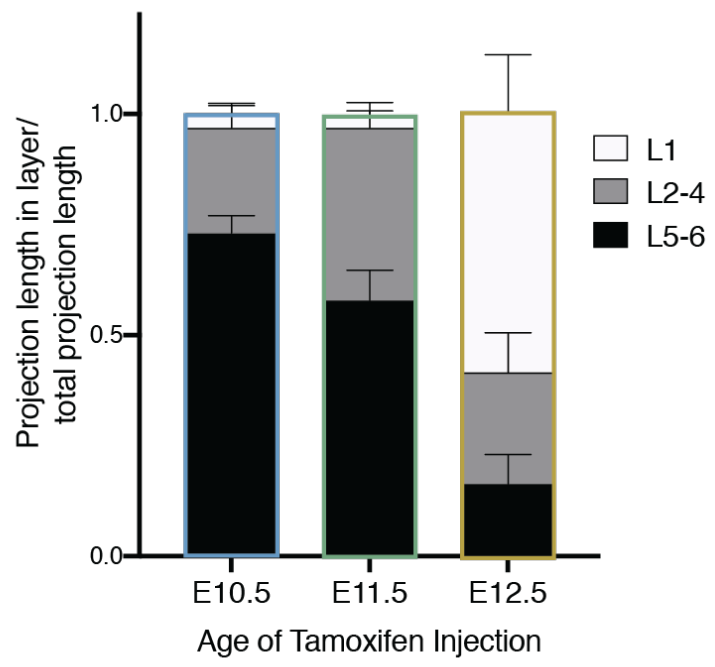

Figure 3 - Figure Supplement 1. Birthdated cholinergic neuron projections to the primary motor and primary visual cortices. (A) Quantification of overall primary motor cortex (M1) innervation at P30 for cholinergic neurons born at each timepoint (One-way ANOVA $(F(3,9)=4.458, p=0.0352)$. (B) Quantification of innervation to specific layers of M1 for each birthdated cohort (Two-way ANOVA $(F(4$, $14)=2.514, p=0.0888)$. (C) Quantification of overall primary visual cortex (V1) innervation at P30 for cholinergic neurons born at each timepoint (One-way ANOVA $(F(3,15)=19.77, p<0.0001)$. (D) Quantification of innervation to specific layers of V1 for each birthdated cohort (Two-way ANOVA $(F(4$, 24) $=12.95, p<0.0001)$. 